\title{
Sur la densité de certains ensembles de multiples, 1
}

\author{
par
}

\section{A. RAOUJ (Marrakech)}

1. Introduction. La motivation du présent travail est la résolution d'une conjecture d'Erdôs sur les ensembles de multiples (l'ensemble des multiples $\mathcal{B}(\mathcal{A})$ d'une suite $\mathcal{A}$ est par définition $\mathcal{B}(\mathcal{A}):=\mathcal{A N})$.

On considère, pour $n$ entier positif, l'ensemble des multiples $\mathcal{B}(n)$ de la suite

$$
\left.\left.\mathcal{D}(n):=\bigcup_{d \mid n}\right] d, 2 d\right] \cap \mathbb{N} .
$$

Erdős a conjecturé que

$$
\mathbf{d} \mathcal{B}(n)=1+o(1) \quad \text { p.p. }
$$

L'argument heuristique suivant constitue la motivation initiale d'Erdős concernant (1.1). Lorsque $n$ et $m$ sont premiers entre eux (hypothèse faite seulement pour simplifier cette argumentation) on sait que le nombre des paires $\{d, t\}$ telles que $d|n, t| m$ et $t \leq 2 n$ peut être minoré par

$$
U_{n, m}=2^{\omega(n)} 2^{\omega(m, n)}, \quad \text { où } \omega(m, n):=\sum_{\substack{p \mid m \\ p \leq n}} 1 .
$$

Le théorème de Hardy et Ramanujan (1917) énonce que pour presque tout entier $n$ on a

$$
\omega(n) \sim \log _{2} n,
$$

et l'on peut montrer similairement que, pour $n$ fixé,

$$
\left|\omega(m, n)-\log _{2} n\right|<\left(\log _{2} n\right)^{2 / 3}
$$

pour tous les entiers $m$ sauf ceux d'une suite de densité tendant vers 0 lorsque $n \rightarrow \infty$. Donc, pour tout entier $n$ d'une suite convenable de densité unité, on a

$$
U_{n, m}=(\log n)^{\log 4+o(1)} \quad \text { p.p. }
$$

En supposant une répartition uniforme des quantités $|\log (t / d)|$ où $t \mid m, t \leq$ $2 n$ et $d \mid n$, on s'attend donc à ce que l'intervalle $\left[0,(\log n)^{-\lambda}\right]$ contienne une 
quote-part de

$$
(\log n)^{\log 4+o(1)}(\log n)^{-\lambda}(\log 2 n)^{-1}
$$

valeurs distinctes $|\log (t / d)|$. La relation (1.1) signifie simplement que cette quote-part est supérieure ou égale à 2 lorsque $\lambda=0$.

En fait, cet argument conduit à une version quantitative de la conjecture initiale, que l'on peut énoncer sous l'une des formes suivantes. Soit $\mathcal{B}_{\lambda}(n)$ l'ensemble des multiples de la suite

$$
\left.\left.\mathcal{D}_{\lambda}(n):=\bigcup_{d \mid n}\right] d,\left(1+(\log n)^{-\lambda}\right) d\right] \cap \mathbb{N} .
$$

(i) Pour $\lambda<\log 4-1$, on a

$$
\mathbf{d} \mathcal{B}_{\lambda}(n)=1+o(1) \quad p . p .
$$

(ii) Pour chaque $0 \leq \lambda<\log 4-1$, il existe une suite $\mathcal{A}$ de densité 1 telle que l'on ait, pour tout $n$ de $\mathcal{A}$ et presque tout $m$,

$$
\begin{aligned}
\mid\left\{(d, t): d|n, t| m, \mu(d t)^{2}=1,|\log (d / t)|\right. & \left.<(\log n)^{-\lambda}\right\} \mid \\
& =(\log n)^{\log 4-1-\lambda+\varepsilon(n)}
\end{aligned}
$$

où $\varepsilon(n) \rightarrow 0$ quand $n \rightarrow \infty$.

Remarque. Soit $\mathcal{A}$ une suite finie d'entiers. Posons

$$
\tau(m, \mathcal{A}):=\sum_{\substack{t \mid m \\ t \in \mathcal{A}}} 1, \quad l(\mathcal{A}):=\sum_{a \in \mathcal{A}} 1 / a
$$

On a

$$
\lim _{X \rightarrow \infty} \frac{1}{X} \sum_{m \leq X} \tau(m, \mathcal{A})=l(\mathcal{A}) .
$$

$\mathrm{Au}$ vu de cette relation, on pourrait être tenté de croire que la densité $\mathbf{d} \mathcal{B}(\mathcal{A})$ est fortement liée à la valeur $l(\mathcal{A})$. Il n'en est rien, en réalité, et la considération des ensembles $\mathcal{D}_{\lambda}(n)$ fournit à cet égard une intéressante classe de contre-exemples. On a

$$
l\left(\mathcal{D}_{\lambda}(n)\right)=(\log n)^{-\lambda+\log 2+o(1)} \quad \text { p.p. }
$$

et

$$
\mathbf{d} \mathcal{B}_{\lambda}(n)=1+o(1) \quad \text { p.p. } \quad(0 \leq \lambda<\log 4-1) .
$$

Cependant, si nous posons pour $0 \leq \lambda<\log 2$,

on a

$$
\begin{aligned}
& \left.\left.\mathcal{A}_{\lambda}^{(1)}(n):=\right] 2, \exp \left\{(\log n)^{-\lambda+\log 2}\right\}\right] \cap \mathbb{N}, \\
& \left.\left.\mathcal{A}_{\lambda}^{(2)}(n):=\right] n \exp \left\{-(\log n)^{-\lambda+\log 2}\right\}, n\right] \cap \mathbb{N},
\end{aligned}
$$

$$
l\left(\mathcal{A}_{\lambda}^{(j)}(n)\right)=(\log n)^{-\lambda+\log 2+o(1)} \quad(j=1,2, n \rightarrow \infty),
$$


alors que le théorème 21 de [HT88] (voir aussi [T84]) implique

$$
\begin{aligned}
& \mathbf{d} \mathcal{B}\left(\mathcal{A}_{\lambda}^{(1)}(n)\right)=1+O\left((\log n)^{\lambda-\log 2}\right), \\
& \mathbf{d} \mathcal{B}\left(\mathcal{A}_{\lambda}^{(2)}(n)\right)=(\log n)^{-\delta(1+\lambda-\log 2)+o(1)} .
\end{aligned}
$$

Il faut encore souligner que la similarité de comportement de $\mathbf{d} \mathcal{B}_{\lambda}(n)$ et $\mathbf{d} \mathcal{B}\left(\mathcal{A}_{\lambda}^{(1)}(n)\right)$ pour $0 \leq \lambda<\log 4-1$ ne provient pas de la présence de "petits" entiers dans $\mathcal{D}_{\lambda}(n)$. En fait, la méthode conduisant à (1.2) montrerait également que l'on a, pour tout $\varepsilon>0$,

$$
\left.\left.\mathbf{d} \mathcal{B}\left(\bigcup_{\substack{d \mid n \\ d>\exp (\log n)^{1-\varepsilon}}}\right] d, 2 d\right] \cap \mathbb{N}\right)=1+o(1) \quad \text { p.p. }
$$

La comparaison de (1.6) et (1.7) fournit donc un exemple de deux suites finies, de mesures logarithmiques voisines, et dont les éléments ont des logarithmes "proches" (c'est-à-dire de la forme $\left.(\log n)^{1+o(1)}\right)$ mais dont les ensembles de multiples ont des densités aussi éloignées que possible asymptotiquement.

Nous renvoyons le lecteur à [R92] pour le contexte dans lequel notre recherche s'insère et pour d'autres détails.

2. Résultats obtenus. Dans ce travail, nous établissons le point (i) de (1.2) sous une forme plus précise et, en fait, on pourrait montrer le point (ii) par la même méthode.

Pour $n$ entier strictement positif et $\lambda$ réel positif ou nul, considérons l'ensemble des multiples $\mathcal{B}_{\lambda}(n)$ de la suite

$$
\left.\left.\mathcal{D}_{\lambda}(n):=\bigcup_{d \mid n}\right] d,\left(1+(\log n)^{-\lambda}\right) d\right] \cap \mathbb{N},
$$

et notons $Q(\alpha):=\alpha \log \alpha-\alpha+1(\alpha>0)$. On a le résultat suivant.

ThÉORÈme 1. Pour $0 \leq \lambda<\lambda^{*}:=\log 4-1$, on $a$

$$
1-e^{-c_{\lambda} \sqrt{\log _{2} n}} \leq \mathbf{d} \mathcal{B}_{\lambda}(n) \leq 1-(\log n)^{-Q(\beta)+o(1)} \quad \text { p.p. }
$$

avec $\beta=((1+\lambda) / \log 2)-1$ et où $c_{\lambda}$ est une constante positive, dépendant uniquement de $\lambda$.

Le théorème 1 sera en fait obtenu en établissant les deux propositions suivantes, qui donnent respectivement la minoration et la majoration de $\mathbf{d} \mathcal{B}_{\lambda}(n)$. On pose

$$
\left.\left.\mathcal{B}_{\lambda}^{\prime}(n):=\mathcal{B}\left(\bigcup_{d \mid n}\right] d,\left(1+(\log d)^{-\lambda}\right) d\right] \cap \mathbb{N}\right),
$$




$$
\nabla(n, m, y):=\sum_{\substack{d|n, t| m \\ 0<\log (t / d) \leq y}} \mu(d t)^{2} .
$$

Proposition 1. Pour tout $0 \leq \lambda<\log 4-1$, il existe $c_{\lambda}>0$ et $x_{0}(\lambda)>1$ tel que l'on ait, pour $X \geq x \geq x_{0}(\lambda)$,

$(2.2)\left|\left\{(n, m) \in[1, x] \times[1, X]: \nabla\left(n, m,(\log n)^{-\lambda}\right)=0\right\}\right| \leq x X e^{-c_{\lambda} \sqrt{\log _{2} x}}$.

Proposition 2. Pour $0 \leq \lambda \leq \log 4-1$, on a

$$
\mathbf{d} \mathcal{B}_{\lambda}^{\prime}(n) \leq 1-(\log n)^{-Q(\beta)+o(1)} \quad \text { p.p. }
$$

avec $\beta=((1+\lambda) / \log 2)-1$.

R e m a r q u e. Une conséquence géométrique simple de (2.2) est la proposition suivante.

Donnons nous au hasard un rectangle de longueur $m$ et de largeur $n$ :

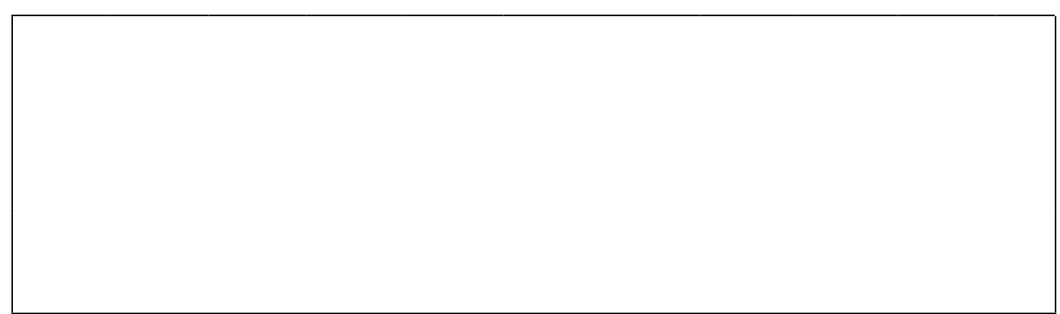

Alors, quitte à retrancher une bande, "relativement petite", de l'une des ses extrémités, la figure admet avec probabilité 1 un quadrillage régulier en carrés d'arête entière $\geq 2$ :

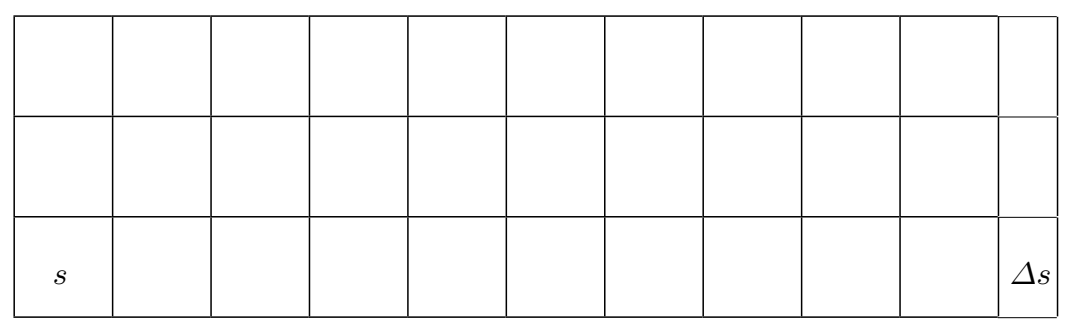

avec

$$
0<\frac{\Delta s}{s} \leq(\log n)^{1-\log 4+\varepsilon} \quad(\varepsilon>0) \quad \text { p.p. }
$$

L'auteur tient à remercier le Professeur G. Tenenbaum qui, par ses conseils et ses critiques, a montré le chemin.

\section{Notations et définitions}

Les lettres $n, m, u, v, i, j, k, l, s$ et $\nu$ désignent des entiers positifs. 
$x, X, y, z, \alpha, \beta, \delta, \lambda, \theta, \eta$ et $\varepsilon$ désignent des réels positifs, $c, c_{0}, c_{1}, \ldots$ sont des constantes absolues positives.

$p, q$ désignent exclusivement des nombres premiers.

$p^{j} \| n$ signifie que $j$ est la valuation $p$-adique de $n$ et $j \neq 0$.

Une somme (resp. produit) portant sur l'ensemble vide est nulle (resp. vaut 1$)$.

$\omega(n)$ est le nombre des facteurs premiers de $n$.

$\Omega(n)$ est le nombre des facteurs premiers de $n$ comptés avec leurs ordres de multiplicité.

$\varphi(n)$ est le nombre des entiers $m \leq n$ qui sont premiers avec $n$.

$\mu(n):= \begin{cases}(-1)^{\omega(n)} & \text { si } n \text { est sans facteur carré, } \\ 0 & \text { sinon. }\end{cases}$

Le plus grand (resp. plus petit) facteur premier de $n$ est noté $P^{+}(n)$ (resp. $P^{-}(n)$ ); par convention : $P^{+}(1)=1, P^{-}(1)=\infty$.

$\Psi(x, y)$ désigne le nombre des entiers $n \leq x$ tels que $P^{+}(n) \leq y$.

$\Phi(x, z)$ désigne le nombre des entiers $n \leq x$ tels que $P^{-}(n)>z$.

$\Theta(x, y, z)$ désigne le nombre des entiers $n \leq x$ tels que $\prod_{p^{j}|| n, p \leq y} p^{j}>z$.

Si $f$ et $g$ sont deux fonctions arithmétiques réels (c'est-à-dire définies de $\mathbb{N}$ dans $\mathbb{R}$ ), alors

$$
f * g(n):=\sum_{d \mid n} f(d) g(n / d) .
$$

Nous introduisons les notations et les définitions suivantes :

$$
\begin{aligned}
n_{k} & :=\prod_{\substack{p \mid n \\
p \leq \exp e^{k}}} p, \quad m(n):=\prod_{p \mid m, p \nmid n} p, \\
\omega(n ; y, z) & :=\sum_{\substack{p^{j} \| n \\
y<p \leq z}} 1, \quad \omega(n, x):=\omega(n ; 1, x), \\
\Omega(n ; y, z): & \sum_{\substack{p^{j} \| n \\
y<p \leq z}} j, \quad \Omega(n, x):=\Omega(n ; 1, x), \\
\tau^{*}(n, \theta) & :=\sum_{d \mid n} \mu(d)^{2} d^{i \theta}, \\
\mathcal{R}(n, \theta) & :=\frac{\left|\tau^{*}(n, \theta)\right|^{2}}{2^{\omega(n)+\omega_{\theta}(n)}} \quad \text { avec } \omega_{\theta}(n):=\omega(n, \exp 1 / \theta), \\
p(n, k, y): & \min \left\{p: p \mid n \text { et } p>\exp \left(y e^{k}\right)\right\} \\
V(u, v, z, \eta) & :=\left|\left\{(d, t): d|u, t| v, \mu(d t)^{2}=1,0<z-\log (d / t) \leq \eta\right\}\right|,
\end{aligned}
$$




$$
\begin{aligned}
& \nabla(u, v, \eta):=V(u, v, 0, \eta)=\sum_{\begin{array}{c}
d|u, t| v \\
0<\log (t / d) \leq \eta
\end{array}} \mu(d t)^{2}, \\
& \left.\left.\mathcal{L}(u, v, \eta):=\bigcup_{\substack{d|u, t| v \\
\mu(d t)^{2}=1}} \log (d / t)+\right] 0, \eta\right] .
\end{aligned}
$$

Pour toute partie $\mathcal{A}$ de $\mathbb{N}$, on note : $\overline{\mathcal{A}}$ le complémentaire de $\mathcal{A}$ dans $\mathbb{N}$ et $\mathbf{1}_{\mathcal{A}}$ la fonction indicatrice de $\mathcal{A}$.

Toute dépendance en fonction d'un paramètre $A$ de $\mathbb{R}^{n}$ des constantes impliquées par les symboles $\ll$ de Vinogradov et $O$ de Landau sera mentionnée par $\ll_{A}$ ou $O_{A}$.

$[x]$ désigne la partie entière de $x$.

$\log _{k}$ est la $k$-ième itérée de la fonction logarithme, définie par

$$
\log _{1} x:=\log x \quad \text { et } \quad \log _{k+1} x:=\log \log _{k} x \quad(k \geq 1) .
$$

\section{Lemmes généraux concernant la répartition des nombres premiers et fonctions arithmétiques}

Lemme 4.1. Pour $0<\varepsilon<1$ et $2<y<z$, on $a$

$$
\begin{aligned}
& \sum_{y<p \leq z} \frac{1}{p^{1-\varepsilon}} \\
& \quad \leq \log \left(\frac{\log z}{\log y}\right)+\int_{0}^{\varepsilon \log z} \frac{e^{x}-1}{x} d x+O\left(z^{\varepsilon} \log z \cdot e^{-\sqrt{\log y}}\right) .
\end{aligned}
$$

Démonstration. D'après une forme forte du théorème des nombres premiers, nous avons

$$
\sum_{y<p \leq z} \frac{1}{p^{1-\varepsilon}}=\int_{y}^{z} \frac{d x}{x^{1-\varepsilon} \log x}+\int_{y}^{z} \frac{1}{x^{1-\varepsilon}} d O\left(x e^{-\sqrt{\log x}}\right) .
$$

L'inégalité (4.1) découle immédiatement de (4.2), en utilisant le changement de variable : $x^{\prime}=\varepsilon \log x$ dans la première intégrale et en intégrant par parties la deuxième.

On applique aussi le théorème des nombres premiers pour obtenir l'évaluation donnée par le lemme suivant.

LEMme 4.2 (voir [T90], p. 381). Soit $f$ une fonction $2 \pi$-périodique, à variation bornée sur $[0,2 \pi]$ et de valeur moyenne

$$
\bar{f}:=\frac{1}{2 \pi} \int_{0}^{2 \pi} f(x) d x
$$


Alors, pour tout triplet $(\theta, w, z)$ de réels tels que $\theta \neq 0$ et $1<w<z$ on a

$$
\begin{aligned}
& \sum_{w<p \leq z} f(\theta \log p) / p \\
= & \bar{f} \log \left(\frac{\log z}{\log w}\right)+O\left\{\left(\frac{V(f)}{|\theta| \log w}\right)+(M(f)(1+|\theta|) V(f)) e^{-\sqrt{\log w}}\right\}
\end{aligned}
$$

où l'on a posé

$$
M(f):=\sup _{x}|f(x)| \quad \text { et } \quad V(f):=\int_{0}^{2 \pi}|d f(x)| .
$$

Le lemme suivant, dû à Halberstam et Richert [HR79] et généralisant un résultat de Hall, nous sera très utile.

Lemme 4.3 (voir [T90]). Soit $f$ une fonction multiplicative, positive et à laquelle on peut associer un couple $\left(\lambda_{1}, \lambda_{2}\right)$ de $\mathbb{R}^{+} \times[0,2[$ tel que pour tout nombre premier $p$ et pour tout entier $j>0$ on ait

$$
f\left(p^{j}\right) \leq \lambda_{1} \lambda_{2}^{j-1} .
$$

Alors, pour tout réel $x \geq 2$,

$$
\begin{aligned}
& \sum_{n \leq x} f(n) \\
& \quad \leq 67\left(1+9 \lambda_{1}+\lambda_{1} \lambda_{2}\left(2-\lambda_{2}\right)^{-2}\right) x \prod_{p \leq x}\left(1-p^{-1}\right) \sum_{j \geq 0} f\left(p^{j}\right) p^{-j} .
\end{aligned}
$$

Nous omettons la démonstration du lemme suivant, qui est banale.

Lemme 4.4. Soit $f$ une fonction arithmétique, multiplicative, telle que $f * \mu \geq 0$. Pour $x \geq 1$, on a

$$
\sum_{n \leq x} f(n) \leq x \prod_{p \leq x}\left(1-p^{-1}\right) \sum_{0 \leq \nu \leq \log x / \log p} f\left(p^{\nu}\right) p^{-\nu} .
$$

Lemme 4.5. Soient $y>1, x>1$. On a

$$
n \varphi(n)^{-1} \leq y
$$

pour tous les entiers $n \leq x$ sauf au plus $\ll x \exp \left(-e^{c_{1} y}\right)$.

D ém o n s t r a t i o n. On applique le lemme précédent à la fonction $f_{a}(n)$ $:=\left(n \varphi(n)^{-1}\right)^{a}, a$ étant un paramètre positif que l'on choisit de manière à minimiser le second membre de l'inégalité

$$
\left|\left\{n: n \leq x, n \varphi(n)^{-1}>y\right\}\right| \leq y^{-a} \sum_{n \leq x} f_{a}(n) .
$$

Les estimations données par les deux lemmes suivants sont des résultats classiques de la théorie du crible. 
Lemme 4.6 (voir [HT88], p. 11). Pour $x \geq 2 z \geq 4$, on a

$$
\Phi(x, z) \asymp \frac{x}{\log z} .
$$

Lemme 4.7 (voir [T90], p. 437). Pour $x \geq z \geq y \geq 2$, on a

$$
\Theta(x, y, z) \ll x \exp \left(-\frac{\log z}{2 \log y}\right) .
$$

5. Lemmes concernant la répartition des facteurs premiers ou des diviseurs. On pose

$$
\omega\left(n_{k}(u)\right):=\sum_{\substack{p \mid n, p \nmid u \\ p \leq \exp e^{k}}} 1, \quad Q(\alpha):=\alpha \log \alpha-\alpha+1 \quad(\alpha>0) .
$$

Lemme 5.1. Soient $\alpha, \delta, u, k$ et $x$ tels que $u \geq 1,0<\alpha<1<\delta \leq 2$ et $1 \leq k \leq \log _{2} x$. On $a$

$$
\alpha k<\omega\left(n_{k}(u)\right)<\delta k
$$

pour tous les entiers $n \leq x$ sauf au plus

$$
\ll x\left(u \varphi(u)^{-1} e^{-Q(\alpha) k}+e^{-Q(\delta) k}\right) .
$$

Démonstration. Le nombre des entiers $n \leq x$ ne vérifiant pas (5.1) ne dépasse pas la quantité

$$
\sum_{n \leq x} \alpha^{\omega\left(n_{k}(u)\right)-\alpha k}+\delta^{\omega\left(n_{k}\right)-\delta k} .
$$

Par application du lemme 4.4 on déduit aisément le resultat souhaité.

Le lemme suivant s'inspire du lemme 51.2 de [HT88] et se démontre de la même manière.

Lemme 5.2. Soient $1 \leq T<k \leq \log _{2} x, u \geq 1$ et $0<\alpha<1$. La minoration

$$
\min _{T \leq s \leq k} \frac{\omega\left(n_{k}(u)\right)-\omega\left(n_{k-s}(u)\right)}{s}>\alpha
$$

est satisfaite pour tous les entiers $n \leq x$ sauf au plus

$$
\ll x u \varphi(u)^{-1} Q(\alpha)^{-1} e^{-T Q(\alpha)} .
$$

Lemme 5.3. Soient $c$ une constante suffisamment grande, $0<\alpha<1$, $x \geq x_{0}(\alpha)$ et $T_{\alpha}=\left[c(1-\alpha)^{-2} \log (2 /(1-\alpha))\right]+1$. Pour tout entier $h$, $T_{\alpha} \leq h \leq \log _{2} x$, et tout ensemble d'entiers $\mathcal{K} \subset\left[h, \log _{2} x\right]$ tel que

(i) $|\mathcal{K}|>c$,

(ii) $\min _{k, k^{\prime} \in \mathcal{K}, k \neq k^{\prime}}\left|k^{\prime}-k\right| \geq h$, 
on $a$

$$
\left|\left\{k \in \mathcal{K}: \min _{T_{\alpha} \leq s \leq h} \omega\left(n_{k}(u) / n_{k-s}(u)\right) / s>\alpha\right\}\right| \geq \frac{9}{10}|\mathcal{K}|
$$

pour tous les entiers $n \leq x$ sauf au plus $x u \varphi(u)^{-1} e^{-|\mathcal{K}|}$.

Démonstration. Posons $T:=T_{\alpha}$. Notons $\sigma_{1}(n)$ le membre de gauche de l'inégalité (5.3) et,

$$
\bar{\sigma}_{1}(n):=|\mathcal{K}|-\sigma_{1}(n) .
$$

Il est clair que l'on a pour tout entier $l \geq 1$

$$
\left|\left\{n: n \leq x, \bar{\sigma}_{1}(n) \geq l\right\}\right| \leq \sum_{n \leq x} \sum_{\substack{\mathcal{H} \subset \mathcal{K} \\|\mathcal{H}|=l}} \prod_{k \in \mathcal{H}} \sum_{T \leq s \leq h} \alpha^{\omega\left(n_{k}(u)\right)-\omega\left(n_{k-s}(u)\right)-\alpha s} .
$$

Intervertissant les signes sommes et produit on a

$$
\begin{aligned}
\mid\{n: n & \left.\leq x, \bar{\sigma}_{1}(n) \geq l\right\} \mid \\
& \leq \sum_{\substack{\mathcal{H} \subset \mathcal{K} \\
|\mathcal{H}|=l}} \sum_{\substack{\left(s_{k}\right) \\
k \in \mathcal{H} \subset\{T, \ldots, h\}^{l}}} \sum_{n \leq x} \prod_{k \in \mathcal{H}} \alpha^{\omega\left(n_{k}(u)\right)-\omega\left(n_{k-s_{k}}(u)\right)-\alpha s_{k}}
\end{aligned}
$$

avec $\left(s_{k}\right)_{k \in \mathcal{H}}:=\left(s_{k_{1}}, \ldots, s_{k_{l}}\right)$ si $\mathcal{H}:=\left\{k_{1}<\ldots<k_{l}\right\}$.

Fixons momentanément $\mathcal{H} \subset \mathcal{K},|\mathcal{H}|=l$ et $\left(s_{k}\right)_{k \in \mathcal{H}} \subset\{T, \ldots, h\}^{l}$. Puisque les intervalles $] k-h, k](k \in \mathcal{H})$ sont deux à deux disjoints, on obtient, en appliquant le lemme 4.3,

$$
\sum_{n \leq x} \prod_{k \in \mathcal{H}} \alpha^{\omega\left(n_{k}(u)\right)-\omega\left(n_{k-s_{k}}(u)\right)} \ll x \exp \left(\sum_{k \in \mathcal{H}} S_{k}+S_{k}^{\prime}\right)
$$

où l'on a posé

$$
\begin{aligned}
& S_{k}:= \sum_{\substack{p \\
k-s_{k}<\log _{2} p \leq k}}(\alpha-1) / p, \\
& S_{k}^{\prime}:=\sum_{\substack{p \mid u \\
k-s_{k}<\log _{2} p \leq k}}(1-\alpha) / p \quad(k \in \mathcal{H}) .
\end{aligned}
$$

Il est clair que $\sum_{k \in \mathcal{H}} S_{k}^{\prime} \leq \log \left(u \varphi(u)^{-1}\right)$ et que $\exp S_{k} \ll \exp \left((\alpha-1) s_{k}\right)$ pour tout $k \in \mathcal{H}$. L'inégalité (5.4) entraîne, par conséquent,

$$
\begin{aligned}
& \left|\left\{n: n \leq x, \bar{\sigma}_{1}(n) \geq l\right\}\right| \\
& \quad \leq c_{3}^{l} x u \varphi(u)^{-1} \sum_{\substack{\mathcal{H} \subset \mathcal{K} \\
|\mathcal{H}|=l}} \sum_{\left(s_{k}\right)_{k \in \mathcal{H}} \subset\{T, \ldots, h\}^{l}} \prod_{k \in \mathcal{H}} e^{-Q(\alpha) s_{k}} \\
& \quad \leq c_{4}^{l} x u \varphi(u)^{-1} 2^{|\mathcal{K}|}\left\{e^{T Q(\alpha)}\left(1-e^{-Q(\alpha)}\right)\right\}^{-l}
\end{aligned}
$$


Cela achève la démonstration puisque pour $T=c(1-\alpha)^{-2} \log (2 /(1-\alpha))$ avec $c$ convenable, on a en particulier $e^{T Q(\alpha)}\left(1-e^{-Q(\alpha)}\right)>c_{4} e^{20}$, de sorte que le dernier membre de (5.5) est majoré par $x u \varphi(u)^{-1} e^{-|\mathcal{K}|}$ pour $l \geq[|\mathcal{K}| / 10]$.

LEMME 5.4. Il existe une constante absolue $x_{0}$ telle que pour $x \geq x_{0}$ et tout ensemble d'entiers $\mathcal{K} \subset\left[3 \log _{3} x, \log _{2} x\right]$ satisfaisant $\grave{a}|\mathcal{K}| \geq x_{0}$ et $\min _{k, k^{\prime} \in \mathcal{K}, k \neq k^{\prime}}\left|k-k^{\prime}\right|>\log |\mathcal{K}|$, on ait

$$
\left|\left\{k \in \mathcal{K}: \log n_{k} \leq 50 e^{k}\right\}\right| \geq \frac{9}{10}|\mathcal{K}|
$$

pour tout les entiers $n \leq x$ sauf au plus $x e^{-|\mathcal{K}|}$.

Démonstration. Soit $l=|\mathcal{K}|$. Notons $k_{1}<\ldots<k_{l}$ les éléments de $\mathcal{K}$. Pour $i \in\{1, \ldots, l\}$, posons

$$
\begin{aligned}
y_{i} & :=e^{k_{i}}, \quad l^{\prime}:=[l / 20], \\
\sigma_{2}(n) & :=\left|\left\{k \in \mathcal{K}: \log n_{k} \leq 50 e^{k}\right\}\right|, \\
\bar{\sigma}_{2}(n) & :=l-\sigma_{2}(n)=\left|\left\{k \in \mathcal{K}: \log n_{k}>50 e^{k}\right\}\right| .
\end{aligned}
$$

Si l'entier $k=k_{i}$ avec $i \geq 2$ de $\mathcal{K}$ est compté dans $\bar{\sigma}_{2}(n)$, on a soit

$$
\log n_{k_{i-1}}>4 l e^{k_{i-1}}
$$

soit

$$
\log n_{k_{i-1}} \leq 4 l e^{k_{i-1}} \quad \text { et } \quad \log n_{k_{i}}>50 e^{k_{i}}
$$

de sorte que l'on peut écrire

$$
\bar{\sigma}_{2}(n) \leq \bar{\sigma}_{2}^{(\mathrm{a})}(n)+\bar{\sigma}_{2}^{(\mathrm{b})}(n)+1
$$

où $\bar{\sigma}_{2}^{(\mathrm{a})}(n)$ (resp. $\left.\bar{\sigma}_{2}^{(\mathrm{b})}(n)\right)$ désigne le nombre des indices $i$ de $\{2, \ldots, l\}$ tels que (a) (resp. (b)) soit réalisé.

Par le lemme 4.7, on a

$$
\sum_{\substack{n \leq x \\ \bar{\sigma}_{2}^{(a)}(n) \geq l^{\prime}}} 1 \leq \frac{1}{l^{\prime}} \sum_{n \leq x} \bar{\sigma}_{2}^{(\mathrm{a})}(n) \leq \frac{1}{l^{\prime}} \sum_{k \in \mathcal{K}} \Theta\left(x, \exp e^{k}, \exp \left(4 l e^{k}\right)\right) \leq x e^{-l-1} .
$$

Par ailleurs, si l'indice $i$ est compté dans $\bar{\sigma}_{2}^{(\mathrm{b})}(n)$, alors

$$
\log \left(n_{k_{i}} / n_{k_{i-1}}\right)>46 e^{k_{i}}
$$

car $k_{i}-k_{i-1}>\log l$. Il suit

$$
\sum_{\substack{n \leq x \\ \bar{\sigma}_{2}^{(\mathrm{b})}(n) \geq l^{\prime}}} 1 \leq \sum_{n \leq x} \sum_{\substack{I \subset\{2, \ldots, l\} \\|I|=l^{\prime}}} e^{-46 l^{\prime}} \prod_{i \in I}\left(n_{k_{i}} / n_{k_{i-1}}\right)^{1 / y_{i}} .
$$

Fixons $I \subset\{2, \ldots, l\}$ avec $|I|=l^{\prime}$, et notons

$$
f(n):=\prod_{i \in I}\left(n_{k_{i}} / n_{k_{i-1}}\right)^{1 / y_{i}} .
$$


Soit $p$ un nombre premier. S'il existe $i \in I$ tel que $\exp e^{k_{i-1}}<p \leq \exp e^{k_{i}}$, alors $f\left(p^{\nu}\right)=f(p)=p^{1 / y_{i}} \leq 3$; sinon $f\left(p^{\nu}\right)=f(p)=1$. Comme en plus la fonction $f$ est multiplicative, alors, par le lemme 4.4, on a

$$
\sum_{n \leq x} f(n) \ll x \prod_{i \in I} \exp \left\{\sum_{k_{i-1}<\log _{2} p \leq k_{i}}\left(\frac{1}{p^{1-1 / y_{i}}}-\frac{1}{p}\right)\right\} .
$$

Par application du lemme 4.1, on a ainsi

$$
\sum_{n \leq x} f(n) \ll x e^{4 l^{\prime}}
$$

Par conséquent,

$$
\sum_{\substack{n \leq x \\ \bar{\sigma}_{2}^{(\mathrm{b})}(n) \geq l^{\prime}}} 1 \leq x 2^{l} e^{-40 l^{\prime}} \leq x e^{-l-1} .
$$

En réunissant (5.7), (5.8) et (5.9) on obtient le lemme 5.4.

Avant d'énoncer le lemme qui suit, rappelons la définition suivante :

$$
p(n, k, y):=\min \left\{p: p \mid n \text { et } p>\exp \left(y e^{k}\right)\right\} .
$$

Lemme 5.5. Soient $x, y$ et $h$ tels que $h \geq x_{0}$ ( $x_{0}$ étant une constante absolue, suffisamment grande) et $1<y<e^{-20-h} \log x$. Soit $\mathcal{K}$ une suite d'entiers de $\left[h, \log _{2} x-\log \left(e^{20} y\right)\right]$ satisfaisant $\grave{a}|\mathcal{K}| \geq x_{0}$ et $\min _{k, k^{\prime} \in \mathcal{K}, k \neq k^{\prime}}\left|k-k^{\prime}\right|$ $\geq 20$. On $a$

$$
\left|\left\{k \in \mathcal{K}: p(n, k, y) \leq \exp \left(y e^{k+20}\right)\right\}\right| \geq \frac{9}{10}|\mathcal{K}|
$$

pour tous les entiers $n \leq x$ sauf au plus $x e^{-|\mathcal{K}|}$.

Démonstration. Posons

$$
\begin{gathered}
l:=[|\mathcal{K}| / 10], \\
\sigma_{3}(n):=\left|\left\{k \in \mathcal{K}: p(n, k, y) \leq \exp \left(y e^{k+20}\right)\right\}\right|, \\
\bar{\sigma}_{3}(n):=|\mathcal{K}|-\sigma_{3}(n),
\end{gathered}
$$

et pour chaque $k \in \mathcal{K}$,

$$
\left.\left.\mathcal{C}_{k}:=\right] \exp \left(y e^{k}\right), \exp \left(y e^{k+20}\right)\right] .
$$

Soit $\chi_{k}$ la fonction multiplicative définie par

Il est clair que

$$
\chi_{k}\left(p^{\nu}\right):= \begin{cases}1 & \text { si } p \notin \mathcal{C}_{k} \\ 0 & \text { sinon. }\end{cases}
$$

$$
\left|\left\{n \leq x: \bar{\sigma}_{3}(n) \geq l\right\}\right| \leq \sum_{n \leq x} \sum_{\substack{\mathcal{H} \subset \mathcal{K} \\|\mathcal{H}|=l}} \prod_{k \in \mathcal{H}} \chi_{k}(n) .
$$


D'après le lemme 4.3 , pour chaque $\mathcal{H} \subset \mathcal{K}$ on a

$$
\sum_{n \leq x} \prod_{k \in \mathcal{H}} \chi_{k}(n) \ll x \exp \sum_{k \in \mathcal{H}} \sum_{p \in \mathcal{C}_{k}}-1 / p
$$

$\operatorname{car} \mathcal{C}_{k} \cap \mathcal{C}_{k}^{\prime}=\emptyset$ si $\left(k, k^{\prime}\right) \in \mathcal{K}^{2}$ et $k \neq k^{\prime}$. On obtient donc, pour $h$ assez grand,

$$
\left|\left\{n \leq x: \bar{\sigma}_{3}(n) \geq l\right\}\right| \ll x 2^{|\mathcal{K}|} e^{-19 l} \leq x e^{-|\mathcal{K}|} .
$$

Dans cette dernière partie du présent paragraphe, nous allons établir des lemmes essentiellement utiles dans la preuve de la proposition 2.

Le résultat combinatoire suivant est un outil important dans l'étude des suites d'entiers $m$ ayant une quantité fixe de facteurs premiers.

Lemme 5.6 (voir [HR66], p. 147). Soient $n \geq 1$ et $\left(x_{1}, \ldots, x_{n}\right) \in \mathbb{R}_{+}^{n}$. Posant, pour chaque entier $k$ de $\{1, \ldots, n\}$,

$$
S_{k}:=\sum_{\substack{I \subset\{1, \ldots, n\} \\|I|=k}} \prod_{i \in I} x_{i}
$$

on $a$

$$
S_{k} \geq\left\{1-\left(\begin{array}{c}
k \\
2
\end{array}\right) \frac{\sum_{i=1}^{n} x_{i}^{2}}{S_{1}^{2}}\right\} \frac{S_{1}^{k}}{k !}
$$

Nous désirons maintenant réserver certaines notations. Soient $n \geq 4$, $\beta \in] 0,1]$ et $U$ une fonction croissant vers l'infini avec

$$
U(n)=\exp (\log n)^{\varepsilon(n)} \quad \text { où } \varepsilon(n) \rightarrow 0 .
$$

Désignons par $\mathcal{M}(n, \beta, U)$ la suite formée par les entiers $m$ tels que

(i) $P^{-}(m)>U(n)$,

(ii) $\Omega(m, n) \leq \beta \log _{2} n$.

Lemme 5.7. Il existe un entier $N(\beta)$ tel que pour tout $n \geq N(\beta)$,

$$
\underline{\mathbf{d}} \mathcal{M}(n, \beta, U) \gg \beta^{-1 / 2}(\log n)^{-Q(\beta)}(\log n)^{\beta \log (1-\varepsilon)}\left(\log _{2} n\right)^{-1 / 2} .
$$

Démonstration. On voit que tout élément $m$ de $\mathcal{M}(n, \beta, U) \backslash\{1\}$ peut s'écrire sous la forme $m=s t$ tels que

- tous les facteurs premiers de $s$ sont dans l'intervalle $] U(n), n]$ et, en comptant leur ordre de multiplicité, leur nombre ne dépasse pas $\beta \log _{2} n$,

- $t$ n'a pas de facteur premier inférieur à $n$.

D'où il ressort que pour $X \geq 1$,

$$
|\mathcal{M}(n, \beta, U) \cap[0, X]| \geq \sum_{1 \leq k \leq \beta \log _{2} n} \sum_{s} \mu(s)^{2} \sum_{\begin{array}{c}
t \leq X / s \\
P^{-}(t)>n
\end{array}} 1 .
$$


Choisissant l'entier $N(\beta)$ aussi grand qu'on veut, considérons $n \geq N(\beta)$ et $X \geq n^{n}$. La dernière somme portant sur $t$ est, d'après le lemme 4.7, minorée (à une constante multiplicative près) par $X /(s \log n)$. Par suite, le lemme 5.6 entraîne

$$
\underline{\mathbf{d}} \mathcal{M}(n, \beta, U) \gg \frac{1}{\log n} \sum_{1 \leq k \leq \beta \log _{2} n} \frac{1}{k !}\left\{\log \left(\frac{\log n}{\log U(n)}\right)+O\left(\frac{1}{\log U(n)}\right)\right\}^{k},
$$

ce qui fournit en particulier

$\underline{\mathbf{d}} \mathcal{M}(n, \beta, U) \gg \frac{1}{\log n} \frac{1}{\left[\beta \log _{2} n\right] !}\left\{\log \left(\frac{\log n}{\log U(n)}\right)+O\left(\frac{1}{\log U(n)}\right)\right\}^{\left[\beta \log _{2} n\right]}$.

Utilisant la formule de Stirling, on a donc pour $n \geq N(\beta)$,

$\underline{\mathbf{d}} \mathcal{M}(n, \beta, U)$

$$
\gg \beta^{-1 / 2}(\log n)^{-Q(\beta)}\left(\log _{2} n\right)^{-1 / 2}\left\{1-\varepsilon+O\left(\frac{1}{\log _{2} n \log U(n)}\right)\right\}^{\beta \log _{2} n}
$$

et le lemme en découle d'une façon claire.

LEMme 5.8. Uniformément pour $s>0,0<\varepsilon_{1}<1 / 2,0<\beta \leq 1$ et $X \geq n \geq 4$, on $a$

$$
\max _{U(n) \leq t \leq n}\left\{\Omega(m, t)-\beta \log \frac{\log t}{\log U(n)}\right\} \leq s
$$

pour tous les entiers $m$ de $\mathcal{M}(n, \beta, U) \cap[0, X]$ sauf au plus

$$
\ll X \beta^{-1} \varepsilon_{1}^{-2}(\log n)^{-Q(\beta)}(\log U(n))^{-\beta}\left(1+\varepsilon_{1}\right)^{-s}\left\{\frac{\log n}{\log U(n)}\right\}^{\beta\left(\varepsilon_{1}-\log \left(1+\varepsilon_{1}\right)\right)} .
$$

Démonstration. Soient $1 \leq z \leq 3 / 2$ et $0<\alpha \leq 1$. Posons

$$
\begin{aligned}
\mathcal{M}:= & \mathcal{M}(n, \beta, U), \quad K:=\left[\log \left(\frac{\log n}{\log U(n)}\right)\right]+1, \\
& t_{k}:=\exp \left(e^{k} \log U(n)\right) \quad(0 \leq k \leq K), \\
E:= & \sum_{\substack{m \in \mathcal{M} \\
m \leq X}}\left\{1: \max _{0 \leq k \leq K}\left(\Omega\left(m, t_{k}\right)-\beta(k-1)\right)>s\right\} .
\end{aligned}
$$

Nous avons

$$
\begin{aligned}
E & \leq z^{-s} \sum_{0 \leq k \leq K} z^{-\beta(k-1)} \sum_{\substack{m \in \mathcal{M} \\
m \leq X}} z^{\Omega\left(m, t_{k}\right)} \\
& \leq z^{-s} \alpha^{-\beta \log _{2} n} \sum_{0 \leq k \leq K} z^{-\beta(k-1} \sum_{\substack{m \leq X \\
P^{-}(m)>U(n)}} z^{\Omega\left(m, t_{k}\right)} \alpha^{\Omega(m, n)} .
\end{aligned}
$$


Cette dernière somme intérieure est, d'après le lemme 4.3,

$$
\ll \frac{X}{\log U(n)}\left(\frac{\log t_{k}}{\log U(n)}\right)^{\alpha z-1}\left(\frac{\log n}{\log t_{k}}\right)^{\alpha-1} .
$$

Donc

$$
E \ll X(\log n)^{\alpha-1-\beta \log \alpha}(\log U(n))^{-\alpha} z^{-s} \sum_{0 \leq k \leq K} e^{k L(\alpha, \beta, z)}
$$

où l'on a posé $L(\alpha, \beta, z):=\alpha z-\alpha-\beta \log z$.

Le choix $\alpha=\beta$ et $z=1+\varepsilon_{1}$ implique alors

$$
L(\alpha, \beta, z)=\left(\varepsilon_{1}-\log \left(1+\varepsilon_{1}\right)\right) \beta
$$

et

$$
\sum_{0 \leq k \leq K} e^{k L(\alpha, \beta, z)} \ll \beta^{-1} \varepsilon_{1}^{-2}\left(\frac{\log n}{\log U(n)}\right)^{\left(\varepsilon_{1}-\log \left(1+\varepsilon_{1}\right)\right) \beta} .
$$

Soit maintenant $t \in] U(n), n]$. Il existe $k(0 \leq k<K)$ tel que $t_{k}<t \leq t_{k+1}$. Donc, si $m$ n'est pas compté dans $E$ alors

$$
\Omega(m, t) \leq \Omega\left(m, t_{k+1}\right) \leq \beta k+s \leq \beta \log \left(\frac{\log t}{\log U(n)}\right)+s .
$$

Notons $\mathcal{M}^{*}=\mathcal{M}^{*}(n, \beta, U)$ la suite formée par les entiers $m$ satisfaisant aux conditions suivantes

$$
\begin{gathered}
P^{-}(m)>U(n), \\
\max _{U(n)<t \leq 3 n} \Omega(m, t) / \log _{2} t \leq \beta .
\end{gathered}
$$

Par le lemme 5.8, nous obtenons le corollaire suivant.

Corollaire 5.1. On a

$$
\begin{aligned}
\underline{\mathbf{d}} \mathcal{M}^{*} \geq & \underline{\mathbf{d}} \mathcal{M}(3 n, \beta, U(n)) \\
& -c_{5} \beta^{-1} \varepsilon_{1}^{-2}(\log n)^{-Q(\beta)}(\log U(n))^{-\beta}(\log n)^{-\beta Q\left(1+\varepsilon_{1}\right)} .
\end{aligned}
$$

Posons

$\nabla_{\lambda}(n, m):=\left|\left\{(d, t): d|n, t| m, \mu(d t)^{2}=1,0<(\log d)^{\lambda} \log (t / d) \leq 1\right\}\right|$.

Lemme 5.9. Uniformément pour $\lambda \geq 0,0<\varepsilon_{1}<1 / 2,0<\beta \leq 1$, $n \geq N(\beta)$ et $X \geq n$, on a

$$
\begin{aligned}
\sum_{\substack{m \in \mathcal{M}^{*} \\
m \leq X}} \nabla_{\lambda}(n, m) \ll & X(\log n)^{-Q(\beta)}(\log U(n))^{-\beta}(\log n)^{\beta \varepsilon_{1} \log 2} \\
& \times(\log U(n))^{-\beta \log 2} \sum_{\substack{d \mid n \\
d>U(n)}}(\log d)^{\beta \log 2-1-\lambda}
\end{aligned}
$$


Démonstration. Posons $\theta(d)=\exp (\log d)^{-\lambda}$ et $s=\beta \varepsilon_{1} \log _{2} n$. Soient $y=y(\beta)$ et $z=z(\beta)$ deux paramètres dans $] 0,1]$. On suppose en outre $\beta \log (1 / z) \leq 1$.

Il est clair que

$$
\begin{aligned}
& \sum_{\substack{m \in \mathcal{M}^{*} \\
m \leq X}} \nabla_{\lambda}(n, m) \sum_{\substack{m \leq X \\
P^{-}(m)>U(n)}} z^{\Omega(m, n)-\beta \log _{2} n} \sum_{d \mid n} \mu(d)^{2} \\
& \sum_{\substack{t \mid m \\
d<t \leq \theta(d) d}} \exp \left\{\left(\Omega(m, t)-\beta \log \left(\frac{\log t}{\log U(n)}\right)-s\right) \log y\right\} \mu(t)^{2} \\
& \ll(\log n)^{-\beta \log z} y^{-s} \sum_{d \mid n} \mu(d)^{2} \\
& \times \sum_{\substack{d<t \leq \theta(d) d \\
P^{-}(t)>U(n)}} \exp \left\{\left(\omega(t)-\beta \log \frac{\log t}{\log U(n)}\right) \log y\right\} z^{\omega(t)} \\
& \times \sum_{\substack{m \leq X / t \\
P^{-}(m)>U(n)}} y^{\Omega(m, t)} z^{\Omega(m, n)}
\end{aligned}
$$

grâce à une interversion de sommations.

Appliquant le lemme 4.3 à la dernière somme portant sur $m$, on obtient

$$
\begin{gathered}
\sum_{\substack{m \in \mathcal{M}^{*} \\
m \leq X}} \nabla_{\lambda}(n, m) \ll X(\log n)^{-\beta \log z-1+z} y^{-s}(\log U(n))^{-z} \\
\times \sum_{\substack{d \mid n \\
d>U(n)}} \mu(d)^{2} \sum_{\substack{d<t \leq \theta(d) d \\
P^{-}(t)>U(n)}} \frac{1}{t}\left(\frac{\log t}{\log U(n)}\right)^{a}(y z)^{\omega(t)}
\end{gathered}
$$

où l'on a posé $a:=y z-z+\beta \log y$.

Par le biais d'une intégration par parties où le lemme 1.4 est intervenu, la dernière somme en $t$ est

$$
\ll \int_{\varrho}^{\varrho+\eta} v^{-b} d v
$$

où l'on a posé

$$
\varrho:=\frac{\log d}{\log U(n)}, \quad \eta:=\frac{1}{(\log d)^{\lambda} \log U(n)} \text { et } b:=1+z-2 y z+\beta \log y .
$$


Si $b>0$, alors la dernière intégrale ne dépasse pas

$$
\eta \varrho^{-b}=(\log d)^{-\lambda-b}(\log U(n))^{b-1} .
$$

Le choix $y=1 / 2$ et $z=\beta$ complète la preuve du lemme 5.9.

Le résultat suivant se démontre de la même manière que le lemme 50.1 de [HT88].

Lemme 5.10. Uniformément pour $x \geq T \geq 2$ et $0<\varepsilon<1 / 2$, on a

$$
\max _{T<z \leq x} \Omega(n, z) / \log _{2} z \leq 1+\varepsilon
$$

pour tous les entiers $n \leq x$ sauf au plus $O\left(x \varepsilon^{-2}(\log T)^{-Q(1+\varepsilon)}\right)$.

Lemme 5.11. Soient $\alpha \in] \log 2,1], x \geq x_{0}(\alpha), \varepsilon=\varepsilon(x) \rightarrow 0$ et $T=$ $T(x) \rightarrow \infty(x \rightarrow \infty)$. On $a$

$$
\sum_{\substack{d \mid n \\ d \geq T}}(\log d)^{-\alpha} \mu(d)^{2} \leq(\log T)^{\log 2-\alpha+\varepsilon}
$$

pour tous les entiers $n \leq x$ sauf au plus

$$
\ll x \varepsilon^{-2}(\log T)^{-\varepsilon^{2} / 2} .
$$

Démonstration. D'une part, d'après le lemme 5.1 on a

$$
\omega(n) \leq(1+\varepsilon) \log _{2} x
$$

pour tous les entiers $n \leq x$ sauf au plus $x(\log x)^{-\varepsilon^{2} / 2}$. Donc

$$
\begin{aligned}
\sum_{\substack{d \mid n \\
d>\sqrt{x}}}(\log d)^{-\alpha} \mu(d)^{2} & \leq(\log \sqrt{x})^{-\alpha} \sum_{\substack{d \mid n \\
d>\sqrt{x}}} \mu(d)^{2} \\
& \leq(\log \sqrt{x})^{-\alpha} 2^{\omega(n)} \leq(\log x)^{-\alpha+\log 2+\varepsilon} .
\end{aligned}
$$

D'autre part, désignant par $S(x)$ la quantité

$$
\sum_{n \leq x} \sum_{\substack{d \mid n \\ T \leq d \leq \sqrt{x}}} \mu(d)^{2}(\log d)^{-\alpha} y^{\Omega(n, d)-(1+\varepsilon) \log _{2} d}
$$

en intervertissant les sommations, on a

$$
S(x) \ll x \sum_{T \leq d \leq \sqrt{x}} \mu(d)^{2}(\log d)^{-\alpha-(1+\varepsilon) \log y} y^{\Omega(d)} d^{-1}(\log d)^{y-1} .
$$

Une simple intégration par parties entraîne ainsi $S(x) \ll x v^{-1}(\log T)^{-v}$ où l'on a posé $v:=1+\alpha+(1+\varepsilon) \log y-2 y$ qu'on suppose $>0$. Le choix $y=1 / 2$ implique $v=\alpha-(1+\varepsilon) \log 2>0$. Par ailleurs, le lemme 5.10 montre que

$$
\max _{T<d \leq n}\left\{\Omega(n, d)-(1+\varepsilon) \log _{2} d\right\} \leq 0
$$


pour tous les entiers $n \leq x$ sauf au plus $\ll x \varepsilon^{-2}(\log T)^{-Q(1+\varepsilon)}$. Donc la quantité

est majorée par

$$
\left|\left\{n \leq x: \sum_{\substack{d \mid n \\ d \geq T}} \mu(d)^{2}(\log d)^{-\alpha}>(\log T)^{\log 2-\alpha+\varepsilon}\right\}\right|
$$

$$
O\left\{x \varepsilon^{-2}(\log T)^{-Q(1+\varepsilon)}\right\}+(\log T)^{\alpha-\varepsilon-\log 2} S(x),
$$

qui est $O\left(x \varepsilon^{-2}(\log T)^{-Q(1+\varepsilon)}\right)$ lorsque $y=1 / 2$.

6. Lemmes concernant les transformées de Fourier des fonctions de répartition liées aux diviseurs. Rappelons tout d'abord les définitions suivantes :

$$
\begin{gathered}
\tau^{*}(n, \theta):=\sum_{d \mid n} \mu(d)^{2} d^{i \theta}, \quad \omega_{\theta}(n):=\sum_{\substack{p \mid n \\
p \leq \exp (1 / \theta)}} 1, \\
\mathcal{R}(n, \theta):=\frac{\left|\tau^{*}(n, \theta)\right|^{2}}{2^{\omega(n)+\omega_{\theta}(n)}} .
\end{gathered}
$$

On voit que

$$
\begin{aligned}
\tau^{*}(n, \theta) & :=2^{\omega(n)}\left(\prod_{p \mid n} p\right)^{i \theta / 2} \prod_{p \mid n} \cos ((\theta \log p) / 2), \\
\mathcal{R}(n, \theta) & :=2^{\omega(n)-\omega_{\theta}(n)} \prod_{p \mid n} \cos ^{2}((\theta \log p) / 2) .
\end{aligned}
$$

Lemme 6.1. Pour $u \geq 1, k \geq 0$ et $\theta$ réel $>0$, on $a$

$$
\sum_{n \leq x} \mathcal{R}\left(n_{k}(u), \theta\right) \ll x u \varphi(u)^{-1}(\log (2+\theta))^{2} .
$$

Démonstration. Si $0<\theta e^{k}<1$, alors pour tout entier $n \geq 1$, on a $\omega_{\theta}\left(n_{k}(u)\right)=\omega\left(n_{k}(u)\right)$, d'où $0 \leq \mathcal{R}\left(n_{k}(u), \theta\right) \leq 1$, ce qui entraîne (6.1).

Supposons $k>\log (1 / \theta)$. Comme conséquence du lemme 4.3, nous avons

avec

$$
\sum_{n \leq x} \mathcal{R}\left(n_{k}(u), \theta\right) \ll x \exp \left(S_{1}+S_{2}+S_{3}\right)
$$

$$
\begin{aligned}
& S_{1}:=\sum_{\substack{p \leq \exp (1 / \theta) \\
p \nmid u}}\left(-1+\cos ^{2}((\theta \log p) / 2)\right) / p, \\
& S_{2}:=\sum_{\substack{\exp (1 / \theta)<p \leq z \\
S_{3}}} \cos (\theta \log p) / p \quad \text { où } z:=\min \left(x, \exp e^{k}\right), \\
& \sum_{p \mid u} 1 / p .
\end{aligned}
$$


On voit clairement que $S_{1} \leq 0$, que $S_{3} \leq \log (u / \varphi(u))$ et que, si $0<\theta<1$ alors, en vertu du lemme 4.2, la somme $S_{2}$ est $O(1)$.

Dans le cas où $\theta \geq 1$, soit $y=y(\theta)$ un paramètre dépendant de $\theta$ tel que $3 \leq y \leq z$. Scindons la somme $S_{2}$ en $S_{21}+S_{22}$, portant respectivement sur les $p \leq y$ et $y<p \leq z$. Il est clair que $S_{21}=\log _{2} y+O(1)$ et que d'après le lemme 4.2,

$$
S_{22}=O\left(1 /(\theta \log y)+(3+\theta) e^{-\sqrt{\log y}}\right) .
$$

Posons $y_{0}:=\exp (\log (2+\theta))^{2}$. Si $z>y_{0}$, alors il découle du choix $y=y_{0}$ l'inégalité

$$
S_{2}=2 \log _{2}(2+\theta)+O(1) .
$$

Par ailleurs, si $z \leq y_{0}$, alors en utilisant le théorème des nombres premiers, on a

$$
S_{2} \leq \sum_{p \leq y_{0}} 1 / p=2 \log _{2}(2+\theta)+O(1) .
$$

En conséquence, nous obtenons

$$
\sum_{n \leq x} \mathcal{R}\left(n_{k}(u), \theta\right) \ll x(u / \varphi(u))(\log (2+\theta))^{2} .
$$

Lemme 6.2. Soient $x, h, \delta, L, \mathcal{K}$ tels que $0<h<\log _{2} x-10, \delta>1$, $L>0$ et $\mathcal{K}$ une suite d'entiers de $\left[h, \log _{2} x\right]$ avec $|\mathcal{K}| \geq 10$. Alors

$$
\left|\left\{k \in \mathcal{K}: \int_{e^{h-k}}^{1} \mathcal{R}\left(n_{k}, \theta\right) \frac{d \theta}{\theta^{\delta}} \leq L(\delta-1)^{-1} e^{(\delta-1)(k-h)}\right\}\right| \geq \frac{9}{10}|\mathcal{K}|
$$

pour tous les entiers $n \leq x$ sauf au plus $O\left(x L^{-1}\right)$.

Démonstration. Posons $l:=[|\mathcal{K}| / 10], \sigma_{5}(n)$ le membre de gauche de l'inégalité $(6.2)$ et $\bar{\sigma}_{5}(n):=|\mathcal{K}|-\sigma_{5}(n)$. Il est clair que

$$
\begin{aligned}
\mid\{n \leq & \left.x: \bar{\sigma}_{5}(n) \geq l\right\} \mid \\
& \leq l^{-1} \sum_{n \leq x} \bar{\sigma}_{5}(n) \leq(\delta-1) l^{-1} L^{-1} \sum_{k \in \mathcal{K}} e^{(1-\delta)(k-h)} \int_{e^{h-k}}^{1} \sum_{n \leq x} \mathcal{R}\left(n_{k}, \theta\right) \frac{d \theta}{\theta^{\delta}} .
\end{aligned}
$$

Le lemme 6.2 découle donc du lemme 6.1. En effet, lorsque $\theta \in] 0,1]$ on a $\sum_{n \leq x} \mathcal{R}\left(n_{k}, \theta\right) \ll x$, d'où

$$
\int_{e^{h-k}}^{1} \sum_{n \leq x} \mathcal{R}\left(n_{k}, \theta\right) \frac{d \theta}{\theta^{\delta}} \ll x(\delta-1)^{-1} e^{-(1-\delta)(k-h)} .
$$

Lemme 6.3. Soient $L>0, H>1$ et $k \geq 0$. On a

$$
\int_{1}^{H} \mathcal{R}\left(n_{k}, \theta\right)(\log (2+\theta))^{2} d \theta \leq L H(\log (2+H))^{4}
$$

pour tous les entiers $n \leq x$ sauf au plus $O\left(x L^{-1}\right)$. 
Démonstration. Le nombre des entiers $n \leq x$ ne vérifiant pas (6.3) est inférieur ou égal à

$$
L^{-1} H^{-1}(\log (2+H))^{-4} \int_{1}^{H}(\log (2+\theta))^{2} \sum_{n \leq x} \mathcal{R}\left(n_{k}, \theta\right) d \theta .
$$

Grâce au lemme 6.1, on voit que cette quantité est

$$
\ll x L^{-1} H^{-1}(\log (2+H))^{-4} \int_{1}^{H}(\log (2+\theta))^{4} d \theta \ll x L^{-1} .
$$

Lemme 6.4. Soient $u \geq 1, N \geq 1$ et $x \geq e^{3}$. Soient $k_{1}<\ldots<k_{N}$ entiers positifs et $\left.\left.\left(\theta_{1}, \ldots, \theta_{N}\right) \in \prod_{i=1}^{N}\right] e^{-k_{i}}, e^{k_{i-1}}\right]$ avec la convention $k_{0}:=$ 0. On a

$$
\sum_{n \leq x} \prod_{i=1}^{N} \mathcal{R}\left(n_{k_{i}}(u), \theta_{i}\right) \leq x c_{6}^{N} u \varphi(u)^{-1} .
$$

Démonstration. Pour chaque entier $k$ et chaque nombre réel $\theta>0$, on a

$$
\begin{aligned}
\mathcal{R}\left(n_{k}(u), \theta\right) & =\prod_{\substack{p \mid n(u) \\
\log p \leq 1 / \theta}} \frac{\left|1+p^{i \theta}\right|^{2}}{4} \prod_{\substack{p \mid n(u) \\
1 / \theta<\log p \leq e^{k}}} \frac{\left|1+p^{i \theta}\right|^{2}}{2} \\
& \leq \prod_{\substack{p \mid n, p \nmid u \\
1 / \theta<\log p \leq e^{k}}}(1+\cos (\theta \log p)) .
\end{aligned}
$$

Comme les intervalles $\left.] 1 / \theta_{i}, e^{k_{i}}\right](1 \leq i \leq N)$ sont deux à deux disjoints dans les conditions de l'énoncé, on peut écrire

$$
\prod_{i=1}^{N} \mathcal{R}\left(n_{k_{i}}(u), \theta_{i}\right) \leq f(n)
$$

où $f$ est la fonction fortement multiplicative définie par

$$
f(p):= \begin{cases}1+\cos \left(\theta_{i} \log p\right) & \left(p \nmid u, 1 / \theta_{i}<\log p \leq e^{k_{i}}\right), \\ 1 & (\text { dans tous les autres cas). }\end{cases}
$$

D'après le lemme 4.3 , on obtient

$$
\sum_{n \leq x} f(x) \ll x \exp \left\{\sum_{p \mid u} 1 / p+\sum_{i=1}^{N} \sum_{\substack{\exp \left(1 / \theta_{i}\right)<p \leq \exp e^{k_{i}} \\ p \leq x}} \cos \left(\theta_{i} \log p\right) / p\right\} .
$$

Par le lemme 4.2, on voit que chaque somme intérieure est $O(1)$. Il suit

$$
\sum_{n \leq x} f(n) \leq c_{6}^{N} u \varphi(u)^{-1} x,
$$

d'où (6.4). 
Lemme 6.5. Soient $x, \delta$ et $\mathcal{K}$ tels que $x \geq x_{0},|\mathcal{K}| \geq x_{0}$ ( $x_{0}$ étant une constante absolue, suffisamment grande), $\mathcal{K}=\left\{k_{1}<k_{2}<\ldots\right\} \subset\left[1, \log _{2} x\right]$ et $\delta>1$. Notons $c_{7}=c_{6} e^{20}$ avec $c_{6}$ la constante apparaissant dans (6.4). Alors

$$
\begin{aligned}
\mid\{i: 1<i \leq|\mathcal{K}|, \\
\left.\quad \int_{e^{-k_{i}}}^{e^{-k_{i-1}}} \mathcal{R}\left(n_{k_{i}}, \theta\right) \frac{d \theta}{\theta^{\delta}} \leq c_{7}(\delta-1)^{-1} e^{(\delta-1) k_{i}}\right\}\left|\geq \frac{9}{10}\right| \mathcal{K} \mid
\end{aligned}
$$

pour tous les entiers $n \leq x$ sauf au plus $x e^{-|\mathcal{K}|}$.

Démonstration. Posons $l:=|\mathcal{K}|, m:=[l / 10]-1, \sigma_{6}(n)$ le membre de gauche de l'inégalité $(6.5), \bar{\sigma}_{6}(n):=l-1-\sigma_{6}(n)$. Il est clair que

$$
\begin{aligned}
\sum_{\substack{n \leq x \\
\bar{\sigma}_{6}(n) \geq m}} 1 \leq \sum_{n \leq x} \sum_{\substack{I \subset\{2, \ldots, l\} \\
|I|=m}} \prod_{i \in I} c_{7}^{-1}(\delta-1) e^{(1-\delta) k_{i}} \int_{e^{-k_{i}}}^{e^{-k_{i}-1}} \mathcal{R}\left(n_{k_{i}}, \theta\right) \frac{d \theta}{\theta^{\delta}} \\
\leq c_{7}^{-m}(\delta-1)^{m} \sum_{\substack{I \subset\{2, \ldots, l\} \\
|I|=m}} \prod_{i \in I} e^{(1-\delta) k_{i}} A(I)
\end{aligned}
$$

où l'on a posé

$$
A(I):=\int_{\left.\left.\Pi_{i} \in I\right] e^{-k_{i}}, e^{-k_{i-1}}\right]}\left\{\sum_{n \leq x} \prod_{i \in I} \mathcal{R}\left(n_{k_{i}}, \theta_{i}\right)\right\} \prod_{i \in I} \frac{d \theta_{i}}{\theta_{i}^{\delta}} .
$$

Utilisant le lemme 6.4, on a

$$
A(I) \leq x c_{6}^{m} \prod_{i \in I} \int_{e^{-k_{i}}}^{e^{-k_{i-1}}} \frac{d \theta}{\theta^{\delta}} \leq x c_{6}^{m}(\delta-1)^{-m} \prod_{i \in I} e^{(\delta-1) k_{i}} .
$$

Il vient donc

$$
\sum_{\substack{n \leq x \\ \bar{\sigma}_{6}(n) \geq m}} 1 \leq x e^{-l}
$$

7. Lemmes sur les mesures d'ensembles d'accroissement. Pour chaque couple d'entiers $(u, v)$ et pour chaque réel positif $\eta$, nous entendons par ensemble d'accroissement l'ensemble

$$
\left.\left.\mathcal{L}(u, v, \eta):=\bigcup_{\substack{d|u, t| v \\ \mu(d t)^{2}=1}} \log (d / t)+\right] 0, \eta\right]
$$

de mesure de Lebesgue $\lambda(u, v, \eta)$. 
Le résultat suivant constitue une étape cruciale dans la démonstration de la proposition 1. Nous faisons appel à une technique d'analyse de Fourier pour l'établir.

Lemme 7.1. Pour $(u, v)=1$ et $\eta>0$, on $a$

$$
\lambda(u, v, \eta) \geq 4^{\omega(u v)}\left(20 \int_{0}^{1 / \eta}\left|\tau^{*}(u v, \theta)\right|^{2} d \theta\right)^{-1} .
$$

Démonstration. Nous commençons par majorer la norme $L^{2}$ de la fonction

$z \rightarrow V(u, v, z, \eta):=\left|\left\{(d, t): d|u, t| v, \mu(d t)^{2}=1,0<z-\log (d / t) \leq \eta\right\}\right|$, lorsque $(u, v)=1$. Désignons par $W$ la fonction définie par

$$
W(y):=\left(\frac{\sin (y / 2)}{y / 2}\right)^{2}
$$

Nous avons $W(y):=\int_{-1}^{1}(1-|\theta|) e^{i \theta y} d \theta$ et $\inf _{0,1]} W=W(1)>0.9$. Nous avons par conséquent, pour tout réel $z$,

$$
\begin{aligned}
V(u, v, z, \eta) & \leq \frac{10}{9} \sum_{d|u, t| v} W\left((z+\log t-\log d) \eta^{-1}\right) \mu(d)^{2} \mu(t)^{2} \\
& \leq \frac{10}{9} \int_{-1}^{1}(1-|\theta|) e^{i \theta z \eta^{-1}} \tau^{*}\left(u,-\theta \eta^{-1}\right) \tau^{*}\left(v, \theta \eta^{-1}\right) d \theta \\
& \leq \frac{10}{9} \eta \int_{-1 / \eta}^{1 / \eta}(1-|\theta| \eta) e^{i \theta z} \tau^{*}(u,-\theta) \tau^{*}(v, \theta) d \theta .
\end{aligned}
$$

La formule de Parseval entraîne donc

$$
\begin{aligned}
\int_{-\infty}^{\infty} V(u, v, z, \eta)^{2} d z & \leq \frac{200}{81} \eta^{2} \pi \int_{-1 / \eta}^{1 / \eta}(1-|\theta| \eta)^{2}\left|\tau^{*}(u,-\theta) \tau^{*}(v, \theta)\right|^{2} d \theta \\
& \leq 20 \eta^{2} \int_{0}^{1 / \eta}\left|\tau^{*}(u v, \theta)\right|^{2} d \theta
\end{aligned}
$$

D'une part, on a

$$
\int_{-\infty}^{\infty} V(u, v, z, \eta) d z=\eta \sum_{d \mid u} \mu(d)^{2} \sum_{t \mid v} \mu(t)^{2}=\eta 2^{\omega(u v)}
$$

et d'après l'inégalité de Cauchy-Schwarz,

$$
\int_{-\infty}^{\infty} V(u, v, z, \eta) d z \leq \sqrt{\lambda(u, v, \eta)}\|V(u, v, \cdot, \eta)\|_{L^{2}(\mathbb{R})} .
$$


On a donc l'inégalité

$$
4^{\omega(u v)} \leq 20 \lambda(u, v, \eta) \int_{0}^{1 / \eta}\left|\tau^{*}(u v, \theta)\right|^{2} d \theta .
$$

Nous sommes maintenant en mesure d'estimer le membre droit de l'inégalité (7.1) lorsque $u=n_{k}, v=m_{k}(n)$ et $\eta=(\log n)^{-\lambda}$. Pour cela, nous commençons par fixer l'entier $n$ dans une suite $\mathcal{A}$ de densité inférieure aussi grande que possible. Nous établissons ensuite une majoration de l'intégrale

$$
\int_{0}^{1 / \eta}\left|\tau^{*}\left(n_{k} m_{k}(n), \theta\right)\right|^{2} d \theta
$$

en écartant un ensemble d'entiers $m$ négligeable.

Nous gardons jusqu'à la fin de ce paragraphe 7 les notations suivantes :

$$
\begin{gathered}
0 \leq \lambda<\log 4-1, \quad \varepsilon=\varepsilon_{\lambda}:=(\log 4-1-\lambda) / 100>0, \quad x \geq x_{0}(\lambda), \\
k_{1}:=\left[(1-3 \varepsilon) \log _{2} x\right], \quad h:=\left[\sqrt{\log _{2} x}\right], \quad l_{0}:=\left[\varepsilon \sqrt{\log _{2} x}\right], \\
\mathcal{K}:=\left\{k_{1}+j h: j=1, \ldots, l_{0}\right\} .
\end{gathered}
$$

Désignons par $\mathcal{A}=\mathcal{A}_{\lambda}(x ; \mathcal{K})$ la suite des entiers $n \leq x$ vérifiant les neuf propriétés suivantes :

$$
\begin{gathered}
n / \varphi(n) \leq \sqrt{\log _{2} x} ; \\
\omega(n) \leq 2 \log _{2} x ; \\
\min _{k \in \mathcal{K}} \min _{h \leq s \leq k} \omega\left(n_{k} / n_{k-s}\right) / s>1-2 \varepsilon ; \\
\left|\left\{k \in \mathcal{K}: \min _{T \leq s \leq h} \omega\left(n_{k} / n_{k-s}\right) / s>1-2 \varepsilon\right\}\right| \geq 9 l_{0} / 10
\end{gathered}
$$

où $T=T(\varepsilon)=c \varepsilon^{-3}, c$ étant une constante absolue suffisamment grande;
$\left|\left\{k \in \mathcal{K}: \log n_{k} \leq 50 e^{k}\right\}\right| \geq 9 l_{0} / 10$
$\left(\mathrm{A}_{6}\right)$

$$
\left|\left\{k \in \mathcal{K}: p(n, k, 100) \leq \exp \left(e^{k+30}\right)\right\}\right| \geq 9 l_{0} / 10
$$
$\left(\mathrm{A}_{7}\right)\left|\left\{k \in \mathcal{K}: \int_{e^{-k}}^{1} \mathcal{R}\left(n_{k}, \theta\right) \frac{d \theta}{\theta^{\delta}} \leq(\delta-1)^{-1} e^{(\delta-1)(k-h / 2)}\right\}\right| \geq 9 l_{0} / 10$

avec $\delta=(1-2 \varepsilon) \log 4$;

$$
\begin{aligned}
& \left(\mathrm{A}_{8}\right) \quad \max _{1 \leq k \leq \log _{2} x} \int_{1}^{(\log x)^{\lambda}} \mathcal{R}\left(n_{k}, \theta\right)(\log (2+\theta))^{2} d \theta \leq(\log x)^{\lambda+2 \varepsilon} \\
& \left(\mathrm{A}_{9}\right) \quad\left|\left\{k \in \mathcal{K}: \int_{e^{-k}}^{e^{-k+h}} \mathcal{R}\left(n_{k}, \theta\right) \frac{d \theta}{\theta^{\delta}} \leq c_{7}(\delta-1)^{-1} e^{(\delta-1) k}\right\}\right| \geq 9 l_{0} / 10
\end{aligned}
$$

où $\delta:=(1-2 \varepsilon) \log 4, c_{7}$ étant la constante figurant dans (6.5). 
D'après les lemmes $4.6,5.1-5.5,6.2,6.3$ et 6.5 où les propriétés $\left(\mathrm{A}_{i}\right)$ $(1 \leq i \leq 9)$ ont été respectivement mises en évidence, nous obtenons le résultat suivant.

LEMME 7.2. On a

$$
\left|\mathcal{A}_{\lambda}(x ; \mathcal{K})\right| \geq x\left(1-e^{-\varepsilon^{2} \sqrt{\log _{2} x}}\right) .
$$

Posons pour $n \in \mathcal{A}_{\lambda}(x ; \mathcal{K}), \mathcal{K}_{n}^{*}$ la suite formée par les $\left[l_{0} / 2\right]$ premiers entiers $k$ qui sont comptés dans $\left(\mathrm{A}_{i}\right)(i=4, \ldots, 7)$ et $\left(\mathrm{A}_{9}\right)$ simultanément.

Lemme 7.3. Soient $n \in \mathcal{A}_{\lambda}(x ; \mathcal{K})$ et $X \geq x$. On a

$$
\max _{k \in \mathcal{K}_{n}^{*}} 4^{-\omega\left(n_{k} m_{k}(n)\right)} e^{k} \int_{1}^{(\log x)^{\lambda}}\left|\tau^{*}\left(n_{k} m_{k}(n), \theta\right)\right|^{2} d \theta \leq 1
$$

pour tous les entiers $m \leq X$ sauf au plus $X(\log x)^{-\varepsilon^{2}}$.

Démonstration. Posons $\alpha:=1-2 \varepsilon$ et $H:=(\log x)^{\lambda}$. Nous savons que

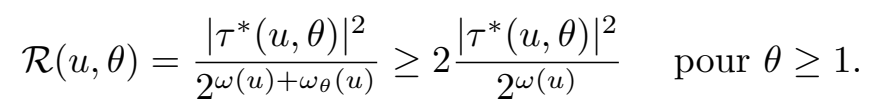

Notant $\overline{\mathcal{C}}_{1}(X)$ le nombre des entiers $m \leq X$ ne vérifiant pas (7.3), on a donc

$$
\begin{aligned}
\overline{\mathcal{C}}_{1}(X) \leq & 4 \sum_{k \in \mathcal{K}_{n}^{*}} e^{k} \int_{1}^{H} \frac{\mathcal{R}\left(n_{k}, \theta\right)}{2^{\omega\left(n_{k}\right)}} \sum_{m \in X} \frac{\mathcal{R}\left(m_{k}(n), \theta\right)}{2^{\omega\left(m_{k}(n)\right)}} d \theta \\
& +\sum_{k_{1}<k \leq \log _{2} x}\left|\left\{m \leq X: \omega\left(m_{k}(n)\right) \leq \alpha k\right\}\right|
\end{aligned}
$$

où l'apostrophe signifie que la somme est portée sur les entiers $m \leq X$ tels que

$$
\min _{k_{1}<k \leq \log _{2} x} \omega\left(m_{k}(n)\right) / k>\alpha .
$$

D'une part, on a

$$
\sum_{m \in X}^{\prime} \frac{\mathcal{R}\left(m_{k}(n), \theta\right)}{2^{\omega\left(m_{k}(n)\right)}} \leq 2^{-\alpha k} \sum_{m \leq X} \mathcal{R}\left(m_{k}(n), \theta\right) \ll X n \varphi(n)^{-1} 2^{-\alpha k}(\log (2+\theta))^{2}
$$

grâce au lemme 6.1. D'autre part, en vertu du lemme 6.1, on a

$$
\sum_{k_{1}<k \leq \log _{2} x}\left|\left\{m \leq X: \omega\left(m_{k}(n)\right) \leq \alpha k\right\}\right| \ll X n \varphi(n)^{-1} e^{-Q(\alpha) k_{1}} .
$$

Par conséquent, on a l'inégalité

$$
\begin{aligned}
\overline{\mathcal{C}}_{1}(X) \ll & X n \varphi(n)^{-1} \\
& \times\left\{Q(\alpha)^{-1} e^{-Q(\alpha) k_{1}}+\sum_{k \in \mathcal{K}_{n}^{*}} e^{k} 4^{-\alpha k} \int_{1}^{H} \mathcal{R}\left(n_{k}, \theta\right)(\log (2+\theta))^{2} d \theta\right\}
\end{aligned}
$$


où nous avons appliqué la minoration $\min _{k \in \mathcal{K}_{n}^{*}} \omega\left(n_{k}\right) / k>\alpha$ donnée par la propriété $\left(\mathrm{A}_{3}\right)$. La propriété $\left(\mathrm{A}_{8}\right)$ implique donc

$$
\begin{aligned}
\overline{\mathcal{C}}_{1}(X) \ll & X n \varphi(n)^{-1} \\
& \times\left\{Q(\alpha)^{-1} e^{-Q(\alpha) k_{1}}+(\log x)^{\lambda+2 \varepsilon} \sum_{k \in \mathcal{K}_{n}^{*}} e^{(1-\alpha \log 4) k}\right\} \\
\ll & X n \varphi(n)^{-1} \\
& \times\left\{Q(\alpha)^{-1} e^{-Q(\alpha) k_{1}}+(\alpha \log 4-1)^{-1}(\log x)^{\lambda+2 \varepsilon} e^{(1-\alpha \log 4) k_{1}}\right\} \\
\ll & X n \varphi(n)^{-1} Q(\alpha)^{-1} e^{-Q(\alpha) k_{1}} \quad\left(k_{1}=\left[(1-3 \varepsilon) \log _{2} x\right]\right) .
\end{aligned}
$$

On obtient donc le lemme 7.3 en utilisant les inégalités $n / \varphi(n) \leq \log _{2} x$ et $Q(1-2 \varepsilon) \geq 2 \varepsilon^{2}$.

Lemme 7.4. Soient $n \in \mathcal{A}_{\lambda}(x ; \mathcal{K}), X \geq x$ et $a_{\varepsilon}=\exp \left(c_{0} \varepsilon^{-3}\right)$ où $c_{0}$ désigne une constante absolue, suffisamment grande. Posant

$$
\sigma(m):=\left|\left\{k \in \mathcal{K}_{n}^{*}: \int_{0}^{1}\left|\tau^{*}\left(n_{k} m_{k}(n), \theta\right)\right|^{2} d \theta \leq 4^{\omega\left(n_{k} m_{k}(n)\right)} a_{\varepsilon} e^{-k}\right\}\right|,
$$

on $a$

$$
\sigma(m) \geq(\varepsilon / 4) \sqrt{\log _{2} x}
$$

pour tous les entiers $m \leq X$ sauf au plus $X e^{-\varepsilon^{2} \sqrt{\log _{2} x}}$.

Démonstration. Notons $\bar{\sigma}(m)=\left[l_{0} / 2\right]-\sigma(m)$. Pour chaque $k \in \mathcal{K}_{n}^{*}$ posons

$$
I_{1, k}:=\left[0, a_{\varepsilon} /\left(3 e^{k}\right)\right], \quad I_{2, k}:=\left[a_{\varepsilon} /\left(3 e^{k}\right), e^{h-k}\right], \quad I_{3, k}:=\left[e^{h-k}, 1\right],
$$

et pour chaque $j \in\{1,2,3\}$,

$$
\mathcal{I}_{j, k}(u, v):=a_{\varepsilon}^{-1} e^{k} 4^{-\omega(u v)} \int_{I_{j, k}}\left|\tau^{*}(u v, \theta)\right|^{2} d \theta .
$$

On a donc

$$
\bar{\sigma}(m)=\left|\left\{k \in \mathcal{K}_{n}^{*}: \sum_{j=1}^{3} \mathcal{I}_{j, k}\left(n_{k}, m_{k}(n)\right)>1\right\}\right| .
$$

Puisque $\mathcal{I}_{1, k}\left(n_{k}, m_{k}(n)\right) \leq 1 / 3$ pour tout $k \in \mathcal{K}_{n}^{*}$, alors, en introduisant

$$
\begin{aligned}
\bar{\sigma}^{\prime}(m) & :=\left|\left\{k \in \mathcal{K}_{n}^{*}: \mathcal{I}_{2, k}\left(n_{k}, m_{k}(n)\right)>1 / 3\right\}\right|, \\
\bar{\sigma}^{\prime \prime}(m) & :=3 \sum_{k \in \mathcal{K}_{n}^{*}} \mathcal{I}_{3, k}\left(n_{k}, m_{k}(n)\right),
\end{aligned}
$$

on a

$$
\bar{\sigma}(m) \leq \bar{\sigma}^{\prime}(m)+\bar{\sigma}^{\prime \prime}(m)
$$


Commençons par majorer $\bar{\sigma}^{\prime \prime}(m)$. L'écriture

$$
\mathcal{I}_{3, k}\left(n_{k}, m_{k}(n)\right)=a_{\varepsilon}^{-1} e^{k} \int_{I_{3, k}} \frac{\mathcal{R}\left(m_{k}(n), \theta\right)}{2^{\omega\left(m_{k}(n)\right)-\omega_{\theta}\left(m_{k}(n)\right)}} \cdot \frac{\mathcal{R}\left(n_{k}, \theta\right)}{2^{\omega\left(n_{k}\right)-\omega_{\theta}\left(n_{k}\right)}} d \theta
$$

montre que la qualité de la majoration de $\mathcal{I}_{3, k}\left(n_{k}, m_{k}(n)\right)$ sera d'autant meilleure que l'on pourra estimer le dénominateur de l'intégrande. Pour cela, faisons appel à la propriété suivante :

$$
\min _{k \in \mathcal{K}_{n}^{*}} \min _{h \leq s \leq k_{1}} \omega\left(m_{k}(n) / m_{k-s}(n)\right) / s>\alpha ;
$$

ici et dans toute cette démonstration $\alpha:=1-2 \varepsilon$.

Nous constatons que si $m$ vérifie (i), alors pour tout $k \in \mathcal{K}_{n}^{*}$ et pour tout réel $\theta \in I_{3, k}$, on a, en posant $s_{k}(\theta):=\left[\log \left(\theta e^{k}\right)\right]$,

$$
\begin{aligned}
\omega\left(m_{k}(n)\right)-\omega_{\theta}\left(m_{k}(n)\right) & \geq \omega\left(m_{k}(n)\right)-\omega\left(m_{k-s_{k}(\theta)}(n)\right) \\
& >\alpha s_{k}(\theta) \geq \alpha \log \left(\theta e^{k}\right)-1 .
\end{aligned}
$$

Dans ce cas, en notant

$$
\mathcal{I}_{3, k}^{\prime}\left(n_{k}, m_{k}(n)\right):=a_{\varepsilon}^{-1} e^{k} 4^{1-\alpha k} \int_{I_{3,4}} \mathcal{R}\left(m_{k}(n), \theta\right) \mathcal{R}\left(n_{k}, \theta\right) \frac{d \theta}{\theta^{\alpha \log 4}},
$$

on a

$$
\mathcal{I}_{3, k}\left(n_{k}, m_{k}(n)\right) \leq \mathcal{I}_{3, k}^{\prime}\left(n_{k}, m_{k}(n)\right)
$$

puisqu'en plus, d'après $\left(\mathrm{A}_{3}\right), n$ vérifie (i).

Or, d'après le lemme 5.2 , le nombre des entiers $m \leq X$ ne vérifiant pas (i) est inférieur à

$$
X l_{0}^{-1} n \varphi(n)^{-1} Q(\alpha)^{-1} e^{-h Q(\alpha)} \leq X\left(\log _{2} x\right)^{-1} e^{-\varepsilon^{2} \sqrt{\log _{2} x}} .
$$

Par conséquent, (7.6) et (7.7) impliquent

$$
\begin{aligned}
\mid\{m \leq X & \left.: \bar{\sigma}^{\prime \prime}(m)>l_{0} / 20\right\} \mid \\
& \leq X\left(\log _{2} x\right)^{-1} e^{-\varepsilon^{2} \sqrt{\log _{2} x}}+60 l_{0}^{-1} \sum_{k \in \mathcal{K}_{n}^{*}} \sum_{m \leq X} \mathcal{I}_{3, k}^{\prime}\left(n_{k}, m_{k}(n)\right) .
\end{aligned}
$$

Appliquant le lemme 6.1 , on a pour $k \in \mathcal{K}_{n}^{*}$,

$$
\sum_{m \leq X} \mathcal{I}_{3, k}^{\prime}\left(n_{k}, m_{k}(n)\right) \ll X n \varphi(n)^{-1} a_{\varepsilon}^{-1} e^{(1-\alpha) k} \int_{e^{h-k}}^{1} \mathcal{R}\left(n_{k}, \theta\right) \frac{d \theta}{\theta^{\alpha \log 4}} .
$$

Nous obtenons donc, en vertu des propriétés $\left(A_{1}\right)$ et $\left(A_{7}\right)$,

$$
\begin{aligned}
\sum_{m \leq X} \mathcal{I}_{3, k}^{\prime}\left(n_{k}, m_{k}(n)\right) & \ll X \sqrt{\log _{2} x} a_{\varepsilon}^{-1} \exp ((1-\alpha \log 4) h / 2) \\
& \leq X\left(\log _{2} x\right)^{-2} e^{-\varepsilon^{2} \sqrt{\log _{2} x}}
\end{aligned}
$$


Il vient donc

$$
\left|\left\{m \leq X: \bar{\sigma}^{\prime \prime}(m)>l_{0} / 20\right\}\right| \leq X\left(\log _{2} x\right)^{-1} e^{-\varepsilon^{2} \sqrt{\log _{2} x}} .
$$

Il nous reste à majorer $\bar{\sigma}^{\prime}(m)$. Pour cela, considérons

$$
\begin{aligned}
\bar{\sigma}_{1}^{\prime}:=\mid\left\{k \in \mathcal{K}_{n}^{*}: \mathcal{I}_{2, k}\left(n_{k}, m_{k}(n)\right)>\right. & 1 / 3 \text { et } \\
& \left.\min _{T \leq s \leq h} \omega\left(m_{k}(n) / m_{k-s}(n)\right) / s>\alpha\right\} \mid
\end{aligned}
$$

avec $T=\left[\log a_{\varepsilon} / 3\right]$. D'après le lemme 5.3 on a

$$
\bar{\sigma}^{\prime}(m) \leq \bar{\sigma}_{1}^{\prime}(m)+\left|\mathcal{K}_{n}^{*}\right| / 10 \leq \bar{\sigma}_{1}^{\prime}(m)+l_{0} / 20
$$

pour tous les entiers $m \leq X$ sauf au plus $X n \varphi(n)^{-1} \exp \left(-\left|\mathcal{K}_{n}^{*}\right|\right)$. Un raisonnement analogue à celui donnant (7.6) implique

$$
\bar{\sigma}_{1}^{\prime}(m) \leq\left|\left\{k \in \mathcal{K}_{n}^{*}: \mathcal{I}_{2, k}^{\prime}\left(n_{k}, m_{k}(n)\right)>1 / 3\right\}\right|
$$

où l'on a posé

$$
\mathcal{I}_{2, k}^{\prime}\left(n_{k}, m_{k}(n)\right):=a_{\varepsilon}^{-1} e^{k} 4^{1-\alpha k} \int_{I_{2, k}} \mathcal{R}\left(m_{k}(n), \theta\right) \mathcal{R}\left(n_{k}, \theta\right) \frac{d \theta}{\theta^{\alpha \log 4}} .
$$

Par conséquent, notant $l=\left[l_{0} / 20\right]$, on a

$$
\begin{aligned}
\left|\left\{m \leq X: \bar{\sigma}_{1}^{\prime}(m)>l\right\}\right| & \leq \sum_{m \leq X} \sum_{\substack{\mathcal{K} \subset \mathcal{K}_{n}^{*} \\
|\mathcal{K}|=l}} \prod_{k \in \mathcal{K}} 3 \mathcal{I}_{2, k}^{\prime}\left(n_{k}, m_{k}(n)\right) \\
& =\sum_{\substack{\mathcal{K} \subset \mathcal{K}_{n}^{*} \\
|\mathcal{K}|=l}} \sum_{m \leq X} \prod_{k \in \mathcal{K}} 3 \mathcal{I}_{2, k}^{\prime}\left(n_{k}, m_{k}(n)\right) .
\end{aligned}
$$

Ecrivant $\prod_{k \in \mathcal{K}} \mathcal{I}_{2, k}^{\prime}\left(n_{k}, m_{k}(n)\right)$ comme intégrale multiple, on a donc

$$
\left|\left\{m \leq X: \bar{\sigma}_{1}^{\prime}(m)>l\right\}\right| \leq 12^{l} a_{\varepsilon}^{-l} \sum_{\substack{\mathcal{K} \subset \mathcal{K}_{n}^{*} \\|\mathcal{K}|=l}} \prod_{k \in \mathcal{K}} e^{(1-\alpha \log 4) k} H(X ; \mathcal{K})
$$

où l'on a posé

$$
H(X ; \mathcal{K}):=\int_{\Pi_{k \in \mathcal{K}} I_{2, k}} \sum_{m \leq X} \prod_{k \in \mathcal{K}} \mathcal{R}\left(m_{k}(n), \theta_{k}\right) \prod_{k \in \mathcal{K}} \mathcal{R}\left(n_{k}, \theta_{k}\right) \prod_{k \in \mathcal{K}} \frac{d \theta_{k}}{\theta_{k}^{\alpha \log 4}} .
$$

Par le lemme 6.4 , on a pour $\mathcal{K} \subset \mathcal{K}_{n}^{*}$ et $|\mathcal{K}|=l$,

$$
\begin{aligned}
H(X ; \mathcal{K}) & \leq X c_{6}^{l} n \varphi(n)^{-1} \prod_{k \in \mathcal{K}} \int_{e^{-k}}^{e^{h-k}} \mathcal{R}\left(n_{k}, \theta\right) \frac{d \theta}{\theta^{\alpha \log 4}} \\
& \leq X c_{8}^{l}(\alpha \log 4-1)^{-l} \prod_{k \in \mathcal{K}} e^{(\alpha \log 4-1) k}
\end{aligned}
$$


où nous avons utilisé les propriétés $\left(\mathrm{A}_{1}\right)$ et $\left(\mathrm{A}_{9}\right)$ de $n$. En reportant la dernière majoration dans (7.10), on obtient

$$
\left|\left\{m \leq X: \bar{\sigma}_{1}^{\prime}(m)>l\right\}\right| \leq X\left(c_{9} / a_{\varepsilon}\right)^{l} \leq X e^{-\sqrt{\log _{2} x}} .
$$

En conséquence, en regroupant (7.5), (7.8), (7.9) et (7.11) on a

$$
\bar{\sigma}(m) \leq \bar{\sigma}^{\prime}(m)+\bar{\sigma}^{\prime \prime}(m) \leq 3\left[\varepsilon \sqrt{\log _{2} x}\right] / 20
$$

pour tous les entiers $m \leq X$ sauf au plus $X e^{-\varepsilon^{2} \sqrt{\log _{2} x}}$.

8. Démonstration de la proposition 1. En s'inspirant de la technique de [MT84] (voir aussi [HT88], Theorem 51) on peut confirmer (1.1) d'une manière non effective. La proposition 1 , qui est plus difficile à établir, est en fait obtenue par une série d'améliorations successives.

Commençons par rappeler et par introduire des notations et des définitions utiles.

Soient $0 \leq \lambda<\log 4-1, \varepsilon=\varepsilon(\lambda)=(\log 4-1-\lambda) / 100>0, X \geq$ $x \geq x_{0}(\lambda), k_{1}:=\left[(1-3 \varepsilon) \log _{2} x\right], l_{0}:=\left[\varepsilon \log _{2} x\right], h:=\left[\sqrt{\log _{2} x}\right]$ et $\mathcal{K}:=$ $\left\{k_{1}+j h: j=1, \ldots, l_{0}\right\}$.

On fixe $n$ dans la suite $\mathcal{A}=\mathcal{A}_{\lambda}(x ; \mathcal{K})$ du lemme 7.2 et on désigne par $k_{1}<\ldots<k_{l_{1}}\left(l_{1}:=\left[l_{0} / 2\right]\right)$ les éléments de $\mathcal{K}_{n}^{*}$.

Soit $i \in\left\{1, \ldots, l_{1}\right\}$. Posons

$$
\begin{gathered}
p_{i}:=\min \left\{p \mid n: p>\exp \left(100 e^{k_{i}}\right\},\right. \\
\mathcal{M}_{i}:=\left\{m \leq X: \nabla\left(n_{k_{i}}, m_{k_{i}}, \eta\right)=0\right\} \quad \text { avec } \eta:=(\log x)^{-\lambda} .
\end{gathered}
$$

Désignons par $\left(\mathrm{a}_{i}\right),\left(\mathrm{b}_{i}\right),\left(\mathrm{c}_{i}\right)$ et $\left(\mathrm{d}_{i}\right)$ les propriétés suivantes pour un entier $m$ :

(a) $\quad \log m_{k_{i}} \leq 50 e^{k_{i}}$.

$\left(\mathrm{b}_{i}\right) \quad \omega\left(m_{k_{i}}\right) \leq 2 k_{i}$.

$\left(c_{i}\right) \quad \lambda\left(n_{k_{i}}, m_{k_{i}}(n), \eta\right) \geq \varepsilon_{1} e^{k_{i}}$ avec $\varepsilon_{1}=\varepsilon_{1}(\lambda)=\exp \left\{-c \varepsilon^{-3}\right\}, c$ étant une constante absolue suffisamment grande.

$\left(\mathrm{d}_{i}\right) \quad$ Il existe au moins un facteur premier $q$ de $m$ tel que $\log q-\log p_{i} \in$ $\mathcal{L}\left(n_{k_{i}}, m_{k_{i}}, \eta\right)$.

Posons

$$
\mathcal{P}_{i}:=\left\{m \leq X: m \text { vérifie }\left(\mathrm{a}_{i}\right),\left(\mathrm{b}_{i}\right) \text { et }\left(\mathrm{c}_{i}\right)\right\} .
$$

Pour chaque $I \subset\left\{1, \ldots, l_{1}\right\}$, notons

$$
\begin{gathered}
\mathcal{P}(I):=\bigcap_{i \in I} \mathcal{P}_{i} \backslash \bigcup_{\substack{1 \leq j \leq \max I \\
j \notin I}} \mathcal{P}_{j}, \\
\mathcal{M}^{\prime}(I):=\mathcal{P}(I) \cap \mathcal{M}_{\max I}, \quad M^{\prime}(I):=\left|\mathcal{M}^{\prime}(I)\right|, \\
\widetilde{\mathcal{M}}(I):=\left\{\widetilde{m}: \text { il existe } m \in \mathcal{M}^{\prime}(I) \text { tel que } \widetilde{m}=m_{k_{\max I}}\right\},
\end{gathered}
$$




$$
\mathcal{I}(l):=\left\{I: I \subset\left\{1, \ldots, l_{1}\right\},|I|=l\right\} .
$$

Fixons momentanément $l \in\left\{1, \ldots,\left[l_{0} / 5\right]\right\}$. Soit $I \in \mathcal{I}(l)$; notons $j:=$ $\max I$. Considérons

$$
\mathcal{F}(I):=\left\{m: m \in \mathcal{M}^{\prime}(I) \text { et } m \text { satisfait }\left(\mathrm{d}_{j}\right)\right\}, \quad F(I):=|\mathcal{F}(I)| .
$$

Remarquons que si $m$ satisfait $\left(\mathrm{d}_{j}\right)$, alors par définition de $\mathcal{L}(u, v)$, il existe un diviseur $d$ de $n_{k_{j}}$ et un diviseur $t$ de $m_{k_{j}}$ tels que $0<\log \left(q t /\left(p_{j} d\right)\right) \leq \eta$; d'où

$$
p_{j} / m_{k_{j}}<q<3 n_{k_{j}} p_{j} .
$$

Par suite, si $m$ satisfait en outre $\left(\mathrm{a}_{j}\right)$, alors en faisant appel aux propriétés $\left(\mathrm{A}_{5}\right)$ et $\left(\mathrm{A}_{6}\right)$ de $\mathcal{A}_{\lambda}(x ; \mathcal{K})$, on a

$$
\sqrt{p_{j}}<q<p_{j}^{2} \quad \text { et } \quad q<\exp \left(2 e^{k_{j}+30}\right)<\exp e^{k_{j+1}} .
$$

Il en découle

$$
\mathcal{F}(I) \subset \mathcal{M}^{\prime}(I) \backslash \mathcal{M}_{j+1} \subset \mathcal{M}^{\prime}(I) \backslash \mathcal{M}^{\prime}(I \cup\{j+1\}) .
$$

En fait, nous avons

$$
\mathcal{F}(I) \subset \mathcal{M}^{\prime}(I) \backslash \bigcup_{J \in \mathcal{I}(l+1)} \mathcal{M}_{J}^{\prime}
$$

En effet, supposons qu'il existe $J \in \mathcal{I}(l+1)$ tel que $\mathcal{F}(I) \cap \mathcal{M}^{\prime}(I) \neq \emptyset$. Comme $\mathcal{F}(I) \subset \mathcal{M}^{\prime}(I) \subset \mathcal{P}(I)$ et $\mathcal{M}^{\prime}(J) \subset \mathcal{P}(J)$, on aurait donc $\mathcal{P}(I) \cap \mathcal{P}(J) \neq \emptyset$; cela implique que $I \subset J$ et $I \neq J$; donc $\mathcal{M}_{\max } J \subset \mathcal{M}_{j+1}$. Notre supposition entraîne alors $\mathcal{F}(I) \cap \mathcal{M}_{j+1} \neq \emptyset$, ce qui contredit (8.2).

Par ailleurs, pour avoir une minoration satisfaisante de $F(I)$, on voit que $\mathcal{F}(I)$ contient tous les entiers $m \leq X$ s'écrivant sous la forme $m=\widetilde{m} t q b$ avec $\widetilde{m} \in \widetilde{\mathcal{M}}(I), t \mid \widetilde{m}, \log q-\log p_{j} \in \mathcal{L}\left(n_{k_{j}}, \widetilde{m}, \eta\right)$ et $P^{-}(b) \geq \exp \left(e^{k_{j}+50}\right) \geq p_{j}^{2}$. Avec les notations $y:=X /(\widetilde{m} t q)$ et $z:=\exp \left(2 e^{k_{j}+30}\right)$, nous avons

$$
F(I) \geq \sum_{\tilde{m}} \sum_{t} \sum_{q} \sum_{b \leq y} 1 .
$$

On vérifie facilement que l'hypothèse $y / 2 \geq z \geq 2$ du lemme 4.6 est satisfaite. D'après ce lemme, nous avons donc

$$
\begin{aligned}
F(I) & \gg X e^{-k_{j}} \sum_{\tilde{m}} 1 / \widetilde{m} \sum_{t \mid \tilde{m}} 1 / t \sum_{q} 1 / q \\
& \gg X e^{-k_{j}} \sum_{\tilde{m}} 1 / \varphi(\widetilde{m}) \sum_{q} 1 / q .
\end{aligned}
$$

Nous sommes par conséquent amenés à minorer $\sum_{q} 1 / q$. On sait que si $\widetilde{m}$ est fixé, alors $q$ est confiné à

$$
\left.\left.\bigcup_{(d, t) \in \mathcal{E}_{j}}\right] d p_{j} / t, e^{\eta} d p_{j} / t\right]
$$


où l'on a posé $\mathcal{E}_{j}:=\left\{(d, t): d\left|n_{k_{j}}, t\right| \widetilde{m},(d, t)=1\right\}$. Remarquons que cette union peut se récrire comme une réunion $\left.\left.\bigcup_{s}\right] y_{s}, z_{s}\right]$ d'au plus $2^{\omega(\tilde{m})+\omega\left(n_{k_{j}}\right)}$ intervalles disjoints. Il est clair que

$$
\sum_{s} \int_{y_{s}}^{z_{s}} d r / r=\lambda\left(n_{k_{j}}, \widetilde{m}(n), \eta\right)
$$

Par ailleurs, d'après (8.1),

$$
\sum_{q} 1 / q \geq \frac{1}{2 \log p_{j}} \sum_{q} \log q / q
$$

Le théorème des nombres premiers permet d'évaluer la dernière somme en $q$ : on a

$$
\sum_{q} \log q / q \geq \lambda\left(n_{k_{j}}, \widetilde{m}(n), \eta\right)+R_{j}
$$

avec

$$
R_{j}:=\sum_{s}\left[\log t \cdot e^{-\sqrt{\log t}}\right]_{y_{s}}^{z_{s}}+\int_{y_{s}}^{z_{s}} O\left(t e^{-\sqrt{\log t}}\right) d(-\log t / t) .
$$

Utilisant $\left.\left(\log y_{s}, \log z_{s}\right) \in\right] \frac{1}{2} \log p_{j}, 2 \log p_{j}\left[2\right.$ et $2^{\omega(\tilde{m})+\omega\left(n_{k_{j}}\right)} \leq 2^{6 k_{j}}$, il vient

$$
R_{j} \ll 2^{6 k_{j}} \log p_{j} \exp \left\{-\left(\frac{1}{2} \log p_{j}\right)^{1 / 2}\right\} \ll \exp \left(-\frac{1}{4} \sqrt{\log p_{j}}\right) .
$$

Par suite, nous obtenons

$$
\sum_{q} 1 / q \gg\left(1 / \log p_{j}\right)\left\{\lambda\left(n_{k_{j}}, \widetilde{m}(n), \eta\right)+O\left(\exp \left(-\frac{1}{4} \sqrt{\log p_{j}}\right)\right)\right\} \gg \varepsilon_{1}
$$

où nous avons fait recours à la propriété $\left(\mathrm{c}_{i}\right)$ de $m$ et à la propriété $\left(\mathrm{A}_{6}\right)$ de $n$. Nous voyons que (8.5) devient ainsi

$$
F(I) \gg X \varepsilon_{1} e^{-k_{j}} \sum_{\tilde{m}} 1 / \varphi(\widetilde{m}) .
$$

Afin de minorer la dernière somme en $\widetilde{m}$, considérons l'inégalité suivante :

$$
M^{\prime}(I) \leq \sum_{\tilde{m}} \sum_{t}^{*} 1
$$

où l'étoile signifie que la somme est portée sur les entiers $t \leq X / \widetilde{m}$ dont chaque facteur premier $q^{\prime}$ vérifie $q^{\prime} \mid \widetilde{m}$ où $q^{\prime}>\exp e^{k_{j}}$. D'après le lemme 4.3 , nous avons

$$
\sum_{t}^{*} 1 \ll X \varphi(\widetilde{m})^{-1} e^{-k_{j}} .
$$

Il en découle

$$
\sum_{\tilde{m}} 1 / \varphi(\widetilde{m}) \gg e^{k_{j}} M^{\prime}(I) / X .
$$


En conséquence, l'inégalité (8.9) devient

$$
F(I) \gg c_{10} \varepsilon_{1} M^{\prime}(I) \text {. }
$$

D'après (8.3) et (8.11), nous avons évidemment

$$
\begin{gathered}
\bigcup_{I \in \mathcal{I}(l)} \mathcal{F}(I) \subset \bigcup_{I \in \mathcal{I}(l)} \mathcal{M}^{\prime}(I) \backslash \bigcup_{J \in \mathcal{I}(l+1)} \mathcal{M}^{\prime}(J), \\
c_{10} \varepsilon_{1} \sum_{I \in \mathcal{I}(l)} M^{\prime}(I) \leq \sum_{I \in \mathcal{I}(l)} F(I) .
\end{gathered}
$$

Puisque

$$
\bigcup_{I \in \mathcal{I}(l)} \mathcal{M}^{\prime}(I) \subset \bigcup_{J \in \mathcal{I}(l+1)} \mathcal{M}^{\prime}(J)
$$

et $\mathcal{M}^{\prime}\left(I^{\prime}\right)=\mathcal{M}^{\prime}\left(I^{\prime \prime}\right)$ pour toute paire $I^{\prime}, I^{\prime \prime}$ de parties de $\left\{1, \ldots, l_{1}\right\}$ telles que $\left|I^{\prime}\right|=\left|I^{\prime \prime}\right|$, la relation (8.12) entraîne clairement

$$
\sum_{I \in \mathcal{I}(l)} F(I) \leq \sum_{I \in \mathcal{I}(l)} M^{\prime}(I)-\sum_{J \in \mathcal{I}(l+1)} M^{\prime}(J) .
$$

Il s'ensuit

$$
\begin{aligned}
\sum_{J \in \mathcal{I}(l+1)} M^{\prime}(J) & \leq\left(1-c_{10} \varepsilon_{1}\right)^{l_{0} / 10} \sum_{I \in \mathcal{I}(1)} M^{\prime}(I) \\
& \leq\left(1-c_{10} \varepsilon_{1}\right)^{l_{0} / 10} M^{\prime}(I) .
\end{aligned}
$$

Remarquons qu'en vertu des lemmes 5.1, 5.4, 7.1, 7.3 et 7.4, nous avons, en posant $l=\left[l_{0} / 10\right]$,

$$
\begin{aligned}
\sum_{J \in \mathcal{I}(l)} M^{\prime}(J) & \geq \sum_{J \in \mathcal{I}(l)}\left|\mathcal{M}_{l_{1}} \cap \mathcal{P}(J)\right| \\
& \geq\left|\mathcal{M}_{l_{1}} \cap \bigcup_{J \in \mathcal{I}(l)} \mathcal{P}(J)\right| \geq M_{l_{1}}-2 X e^{-\varepsilon^{2} \sqrt{\log _{2} x}}
\end{aligned}
$$

Il découle donc de (8.15) et (8.16)

$$
M_{l_{1}} \leq X\left(2 e^{-\varepsilon^{2} \sqrt{\log _{2} x}}+\left(1-c_{10} \varepsilon_{1}\right)^{l_{0} / 10}\right) \leq X \exp \left(-\varepsilon_{2} \sqrt{\log _{2} x}\right)
$$

avec $\varepsilon_{2}=\varepsilon_{2}(\lambda):=\exp \left(-c_{11} \varepsilon^{-3}\right)$. On obtient enfin, en appliquant le lemme 7.2,

$$
\begin{aligned}
\sum_{n \leq x} \sum_{m \leq X}\{1: \nabla(n, m, \eta)=0\} & \leq x X e^{-\varepsilon^{2} \sqrt{\log _{2} x}}+\sum_{n \in \mathcal{A}_{\lambda}(x ; \mathcal{K})} M_{l_{1}} \\
& \leq 2 x X \exp \left(-\varepsilon_{2} \sqrt{\log _{2} x}\right) .
\end{aligned}
$$

Cela achève la démonstration de la proposition 1. 
9. Démonstration de la proposition 2. Soit $\lambda \in[0, \log 4-1]$ fixé. Notre objectif est de minorer $1-\mathbf{d} \mathcal{B}_{\lambda}^{\prime}(n)$ p.p. Pour cela, considérons

$$
\begin{gathered}
\varepsilon=\varepsilon(n):=\left(\log _{2} n\right)^{-1 / 6}, \quad \varepsilon_{1}=\varepsilon_{1}(n):=\varepsilon+2 \varepsilon^{2}, \\
U=U(n):=\exp (\log n)^{\varepsilon(n)}, \quad \beta:=\left(\frac{1+\lambda-2 \varepsilon}{\log 2}-1\right) \frac{\varepsilon}{\varepsilon_{1}}, \\
\mathcal{N}_{\lambda}:=\left\{n \geq N(\lambda): \sum_{\substack{d \mid n \\
d>U}}(\log d)^{-\alpha} \leq(\log U)^{-\alpha+\log 2+\varepsilon}\right\},
\end{gathered}
$$

où l'on a posé $\alpha:=\beta \log 2-1-\lambda$. D'après le lemme 5.11 , on a

$$
\mathrm{d} \mathcal{N}_{\lambda}=1 \text {. }
$$

Soit $\mathcal{M}^{*}$ la suite formée par les entiers $m$ satisfaisant aux conditions suivantes :

$$
\begin{gathered}
P^{-}(m)>U(n), \\
\max _{U(n)<t \leq 3 n} \Omega(m, t) / \log _{2} t \leq \beta .
\end{gathered}
$$

D'après le lemme 5.7 et le corollaire 5.1 , il est facile de voir que

$$
\underline{\mathbf{d}} \mathcal{M}^{*} \gg \beta^{-1 / 2}(\log n)^{-Q(\beta)}(\log n)^{\beta \log (1-\varepsilon)}\left(\log _{2} n\right)^{-1 / 2} .
$$

Par ailleurs, si

$$
\nabla_{\lambda}(n, m):=\left|\left\{(d, t): d|n, t| m, \mu(d t)^{2}=1,0<(\log d)^{\lambda} \log (d / t) \leq 1\right\}\right|,
$$

alors par le lemme 5.9 , on a

$$
\lim _{X \rightarrow \infty} \sup \frac{1}{X} \sum_{\substack{m \in \mathcal{M}^{*} \\ m \leq X}} \nabla_{\lambda}(n, m) \ll(\log n)^{-Q(\beta)}(\log U)^{-\beta}(\log n)^{-r}
$$

où l'on a posé $r=(\lambda+1-\log 2-\varepsilon) \varepsilon-\beta \varepsilon_{1} \log 2$. Il en résulte en particulier

$$
\lim _{X \rightarrow \infty} \sup \frac{1}{X} \sum_{\substack{m \in \mathcal{M}^{*} \\ m \leq X}} \nabla_{\lambda}(n, m)=o\left(\underline{\mathbf{d}} \mathcal{M}^{*}\right) .
$$

En conséquence, l'inégalité

$$
\begin{aligned}
\mid\{m \leq X: m \in & \left.\mathcal{B}_{\lambda}^{\prime}(n)\right\} \mid \\
& \leq\left|\left\{m \leq X: m \notin \mathcal{M}^{*}\right\}\right|+\left|\left\{m \leq X: m \in \mathcal{M}^{*} \cap \mathcal{B}_{\lambda}^{\prime}(n)\right\}\right|
\end{aligned}
$$

entraîne, grâce à (9.1)-(9.3),

$$
1-\mathbf{d} \mathcal{B}_{\lambda}^{\prime}(n) \geq(\log n)^{-Q(\beta)+o(1)} \quad \text { p.p. }
$$

$\operatorname{avec} \beta=(1+\lambda) / \log 2-1$. 


\section{Bibliographie}

[HR79] H. Halberstam and H.-E. Richert, On a result of R. R. Hall, J. Number Theory 11 (1979), 76-89.

[HR66] H. Halberstam and K. F. Roth, Sequences, Oxford University Press, 1966.

[HT88] R. R. Hall and G. Tenenbaum, Divisors, Cambridge University Press, 1988.

[MT84] H. Maier and G. Tenenbaum, On the set of divisors of an integer, Invent. Math. 76 (1984), 121-128.

[R92] A. Raouj, Sur la densité de certains ensembles de multiples, Thèse de l'Université de Nancy I, 1992.

[T84] G. Tenenbaum, Sur la probabilité qu'un entier possède un diviseur dans un intervalle donné, Compositio Math. 51 (1984), 243-263.

[T90] —, Introduction à la théorie analytique et probabiliste des nombres, Publications de l'Institut Elie Cartan, Université de Nancy I, 1990.

UNIVERSITÉ CADI-AYYAD

FACULTÉ DES SCIENCES, SEMLALIA

DÉPARTEMENT DE MATHÉMATIQUES

B.P. S.15, MARRAKECH, MAROC 